\title{
Flight Envelope Discovery for Damage Resilience with Application to an F-16
}

\author{
Heejun Choi* and Ella M. Atkins ${ }^{\dagger}$ \\ University of Michigan, Ann Arbor, MI 48105 \\ Guoxing $\mathrm{Yi}^{\ddagger}$ \\ Harbin Institute of Technology, Harbin, Heilongjiang, 150001, China
}

\begin{abstract}
Safe aircraft emergency management requires a two-stage real-time response: first identify the problem, then alter the flight plan and constrain effector commands to maximize chances of a safe landing. In cases of degraded aircraft performance, commands must respect new flight envelope limits to avoid loss-of-control. In previous work we have proposed an intuitive approach to adaptive flight planning and guidance in which feasible trim states are sequenced into emergency landing flight plans. Sets of feasible trim states and transitions between these states are derived from post-failure aircraft dynamics, enabling the flight planner to focus on real-time generation of landing trajectory geometry (kinematics) without risk of violating dynamics constraints. This paper integrates the guidance and planning algorithms required to autonomously discover the flight envelope and then to plan a path (in real-time) to a nearby landing runway. Our method accommodates nontraditional flight envelope constraints, including cases in which the aircraft cannot maintain straight and/or level flight. Two F-16 control surface jam failure cases are used to illustrate algorithm integration and performance.
\end{abstract}

\section{Nomenclature}

$\begin{array}{ll}\text { APF } & \text { Artificial Potential Field } \\ \text { TSD } & \text { Trim State Discovery } \\ \text { TDV } & \text { Turning Dubins Vehicle } \\ \mathbf{z} & \text { Aircraft state vector } \\ \mu & \text { Actuation vector } \\ x, y, z & \text { Aircraft 3-D position (sub-vector of } \mathbf{z} \text { ) } \\ U, V, W & \text { Aircraft velocity vector (sub-vector of } \mathbf{z} \text { ) } \\ \phi, \theta, \psi & \text { Aircraft orientation as Euler angles (sub-vector of } \mathbf{z} \text { ) } \\ p, q, r & \text { Aircraft angular rates (sub-vector of } \mathbf{z} \text { ) } \\ \alpha & \text { Angle of attack } \\ \beta & \text { Sideslip angle } \\ \gamma & \text { Flight path angle } \\ V_{T} & \text { True airspeed } \\ \dot{\psi} & \text { Turn rate } \\ \dot{h} & \text { Climb rate } \\ \vec{F}_{A} & \text { Attractive force for the TSD APF } \\ \vec{F}_{P} & \text { Repulsive force for the TSD APF } \\ \vec{F}_{R} & \text { Resultant (total) force for the TSD APF } \\ \mathbb{T} & \text { Path in trim state space for TSD } \\ \sigma & \text { Curve (Lateral Plane Landing Path) for the TDV }\end{array}$

*Research Assistant, Aerospace Engineering, University of Michigan, Ann Arbor, MI 48105

$\dagger$ Associate Professor, Aerospace Engineering, University of Michigan, Ann Arbor, MI 48105, Associate Fellow

$\ddagger$ Associate Professor, Control Science and Engineering Dept., Harbin Institute of Technology, Harbin, China 


\begin{tabular}{|c|c|}
\hline $\mathcal{O}$ & Circular curve for the TDV \\
\hline$a$ & Circular arc curve for the TDV \\
\hline$b$ & Product of circular arc curves \\
\hline$\Sigma$ & Set of possible curves for the TDV \\
\hline$\Sigma_{c}$ & Set of circular curves for the TDV \\
\hline $\mathcal{A}$ & Set of circular arc curves for the TDV \\
\hline $\mathcal{A}_{r}$ & Set of possible reference arcs connecting two centers of the initial and final circular curves \\
\hline $\mathcal{B}$ & Set of possible sequences of two different turning radii for the TDV \\
\hline $\mathcal{C}$ & Natural representation of $\sigma$ with respect to a center $c_{1}$ of the first circular curve for the TDV \\
\hline$s$ & Length of the arc segment of the $\sigma$ \\
\hline$\vec{V}$ & Velocity vector \\
\hline$\vec{T}$ & Unit tangent with respect to $c_{1}$ \\
\hline$\vec{k}$ & Curvature vector with respect to $c_{1}$ \\
\hline$\vec{n}$ & Principal normal unit vector with respect to $c_{1}$ \\
\hline$\vec{b}$ & Unit binormal vector with respect to $c_{1}$ \\
\hline$r$ & Radius of circular curve \\
\hline$\delta$ & Central angle of the reference arc \\
\hline$l$ & Length of flight path arc curves \\
\hline $\mathcal{J}$ & Length of the reference arc traversed over a two-arc sequence \\
\hline$n$ & Number of arc sequences in $\mathcal{B}$ \\
\hline$n_{m}$ & Minimum number of arc sequences in $\mathcal{B}$ \\
\hline$\lambda$ & Distance of the points on a straight line from a known point \\
\hline$h_{0}$ & Initial altitude of the TDV \\
\hline \multicolumn{2}{|c|}{ Subscript } \\
\hline$r$ & Reference arc \\
\hline 1 & First circular curve of the TDV alternating arc sequence \\
\hline 2 & Second circular curve of the TDV alternative arc sequence \\
\hline$m$ & Minimum radius turning circle \\
\hline$M$ & Maximum radius turning circle \\
\hline $\mathcal{T}$ & Trim state \\
\hline
\end{tabular}

\section{Introduction}

Flight vehicles ranging from unmanned aicraft systems (UAS) to commercial transport are becoming increasingly reliable. With triply-redundant components and systems, safety-critical commercial transport electro-mechanical failures are increasingly rare. Loss-of-control, however, remains the most common factor identified in accident investigations, with causes ranging from loss of pilot situational awareness to component or system failure. UAS do not and cannot given cost/weight constraints be equipped with triple or even dual redundancy, but UAS typically have far more flexibility to safely land in a small clear area should a problem arise.

For any flight vehicle, the top safety-oriented goal in an emergency is to land without incident. A safe landing requires the accomplishment of two sequential tasks. First, the impact of the anomaly on aircraft handling qualities and/or situational awareness must be sufficiently characterized to support a safe landing. Next, the landing plan that respects reduced performance constraints must be devised and safely executed. This process represents a number of challenges traditionally left to the human pilot. The autopilot must avoid loss-of-control by establishing and maintaining a stabilizable flight condition. System identification technology can assist with updating pertinent performance models. Changes in performance are of use to both the automation and the flight crew, and potentially of use to neighboring air traffic and ground-based controllers. Finally, a safe landing flight plan must be generated and followed.

We have studied the problem of flight planning and guidance for safe emergency landing in the context of failures and damage that degrade aircraft performance. ${ }^{1-5}$ Situations of this class include engine or actuator failures (jams) or structural damage due to cuases such as fatigue, attack, or collision. In our initial work ${ }^{1}$ we proposed an adaptive flight planning architecture that generated a flight plan by first selecting a landing 
runway then building a landing trajectory to that runway that met flight envelope constraints. Landing site is identified in under a second on a single-core desktop PC by efficiently defining a footprint, identifying runways within that footprint, downselecting to runways that meet minimum constraints such as length, wind, and instrument approach, then utilizing a multi-objective utility function to prioritize these runways. A Dubins path is then generated to this landing runway, requiring a sequence of stabilizable trim states capable of turning and straight flight in a shallow descent. Initial work ${ }^{1}$ examined applicability of adaptive flight planning to loss-of-thrust scenarios, also extensible to cases in which an onboard emergency (e.g., pilot incapacitation) results in a need to land with nominal performance characteristics. Our work was then extended to analysis of control surface jams, ${ }^{2,3}$ resulting in identification of F-16 flight envelope databases for control surface jams referenced in this paper. In Strube et al, ${ }^{2,3}$ a general search-based approach to trim state sequencing was proposed to generate landing trajectories for arbitrary failure cases. This method, however, would be difficult to certify given the combinatorial nature of the search space resulting in exponential worst-case complexity.

Figure 1 illustrates the emergency flight management architecture providing context for the adaptive flight planner (AFP) studied in this work. A Flight Plan Monitor continually validates the existing flight plan against the most current system model to verify feasibility of the flight plan. If the executing flight plan becomes infeasible, the pilot is notified via the Pilot Interface. Concurrently, the AFP is activated to generate a new flight plan. Within the AFP, a Landing Site Search (LSS) module identifies a safe landing site, currently defined as a runway deemed safe based on the degraded aircraft performance model. The Segmented Trajectory Planner then constructs a dynamically feasible trajectory to the landing site. The Segmented Trajectory Planner relies on the feasible and stabilizable post-failure/damage trim flight states as building blocks of a segmented landing trajectory. In this paper, we rely on two real-time analytic trajectory planners: the Dubins-based planner summarized above when possible and a Turning Dubins Vehicle (TDV) planner first introduced in Choi and Atkins ${ }^{5}$ otherwise. Both build trajectories based on the feasible postfailure flight envelope, from which constraints on turn rate, flight path angle, and airspeed are derived. In Yi and Atkins, ${ }^{6}$ a trim state discovery (TSD) algorithm was introduced to guide the flight planner through the space of trim states advantageous for landing.

This paper is the first publication in which trim state discovery and real-time trajectory planning algorithms are integrated in an end-to-end flight planning and guidance system. Below, we first review our model of trim states and flight envelope, then summarize the trim state discovery (TSD) algorithm integrated into this work. Next, we review the Turning Dubins Vehicle (TDV) algorithm and present a nontrivial extension to a three-dimensional solution that accounts for flight path angle constraints. We present a series of flight envelope profiles for an F-16 with jammed aileron and rudder to illustrate trim state database properties and to highlight the manner in which database storage can be minimized through exploitation of flight envelope overlap between jam angles. We then present Dubins and TDV landing trajectories over a series of limited TSD-generated local envelopes to illustrate both the utility of the algorithms and the impact of limited TSD on landing solutions. We conclude with a summary of findings and future work to mature this technology to a form where it can ultimately be certified for use in manned and unmanned aircraft.

\section{Trim State}

We assume a flat earth model and adopt a rigid body model of aircraft dynamics and kinematics similar to that presented in Stevens and Lewis. ${ }^{7}$ The state vector includes the components $\left[\begin{array}{ccc}x & y & z\end{array}\right]^{T}$ of the inertial position vector, elements $\left[\begin{array}{lll}U & V & W\end{array}\right]^{T}$ of the translational velocity vector $\vec{v}_{B}$, Euler angles $\left[\begin{array}{lll}\phi & \theta & \psi\end{array}\right]^{T}$, and angular rates $\left[\begin{array}{ccc}p & q & r\end{array}\right]^{T}$. Since the aerodynamic force and moment components depend on the aerodynamic angles and the true airspeed, we replace state variables $U, V$, and $W$ by true airspeed $V_{T}$, angle of attack $\alpha$, and sideslip angle $\beta$. Then state vector $\mathbf{z}$ is given by:

$$
\mathbf{z}=\left[\begin{array}{llllllllllll}
V_{T} & \alpha & \beta & p & q & r & x & y & z & \phi & \theta & \psi
\end{array}\right]^{T}
$$

Actuation vector $\mu$ is given by:

$$
\mu=\left[\begin{array}{llll}
\mu_{t} & \mu_{e} & \mu_{a} & \mu_{r}
\end{array}\right]^{T}
$$




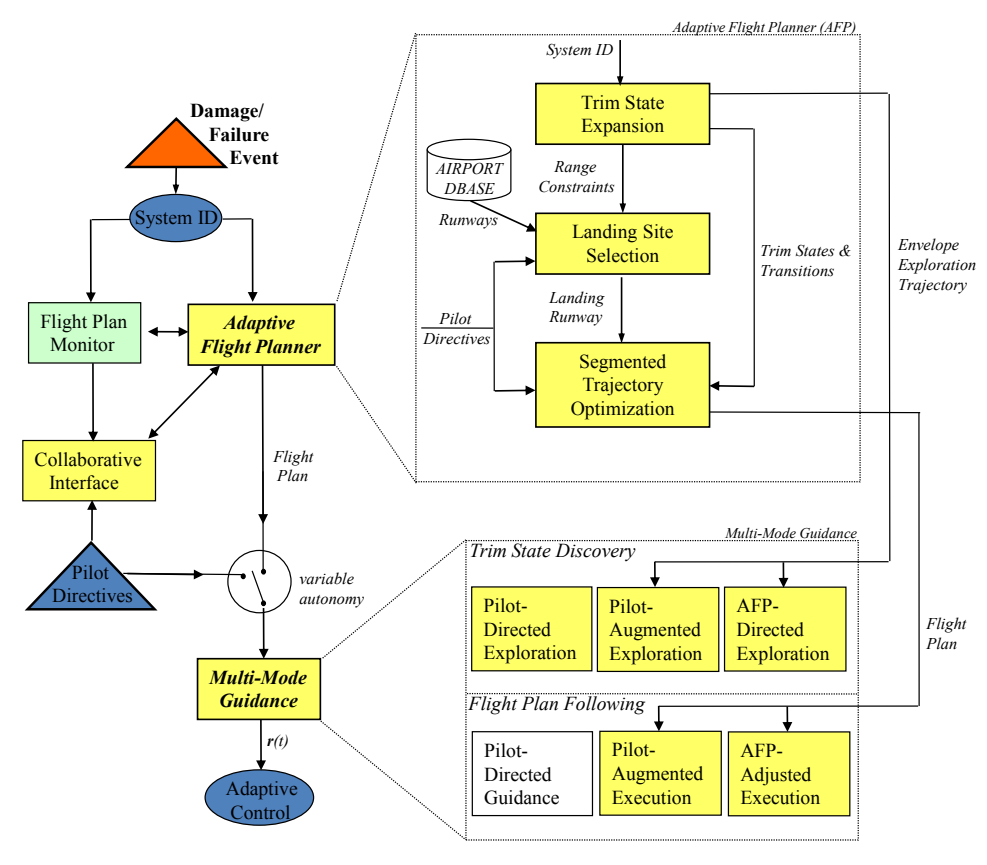

Figure 1. Emergency Flight Management Architecture.

where $\mu_{t}, \mu_{e}, \mu_{a}$, and $\mu_{r}$ are engine throttle, elevator, aileron, and rudder control commands, respectively. The standard 6-DOF equations of motion for a rigid-body aircraft used to describe the F-16 aircraft are then given by:

$$
\dot{\mathbf{z}}=f(\mathbf{z}, \mu)
$$

where $f$ is a vector of twelve scalar nonlinear functions.

A trim state is defined as an unaccelerated or equilibrium state. We use trim states as a discrete approximation of the stable or stabilizable aircraft flight envelope, defining nominal and post-failure feasible flight conditions. Formally, a trim state is a condition in which:

$$
\frac{d}{d t}\left[\begin{array}{llllll}
V_{T} & \alpha & \beta & p & q & r
\end{array}\right]=0
$$

Both Dubins vehicle and Turning Dubins Vehicle trajectories constrain an aircraft to move along path segments with constant curvature bounded above and below. ${ }^{5,8}$ Augmented with a steady climbing/descending flight path angle, our landing trajectories are defined as sequences of level or climbing/descending straight or turning flight segments consistent with Dubins or TDV geometries and defined by:

$$
\begin{aligned}
& {\left[\begin{array}{llllll}
\dot{V}_{T} & \dot{\alpha} & \dot{\beta} & \dot{p} & \dot{q} & \dot{r}
\end{array}\right]=0} \\
& {\left[\begin{array}{ll}
\dot{\phi} & \dot{\theta}
\end{array}\right]=0} \\
& \dot{\psi}=\dot{\psi}^{*} \\
& \dot{h}=\dot{h}^{*}
\end{aligned}
$$

where $\dot{\psi}^{*}$ and $\dot{h}^{*}$ are the desired constant turn rate and climb rate for the trim condition. The climbing/descending turning flight includes straight and level flight, straight climbing/desceding flight, and level turning flight. For example, straight and level flight is a special case of climbing/descending turning flight if $\dot{\psi}=0$ and $\dot{h}=0$. From Eq. 5, a trim state can be fully defined via the reduced state vector

$$
\overline{\mathbf{z}}=\left[\begin{array}{llllllll}
V_{r} & \alpha & \beta & p & q & r & \phi & \theta
\end{array}\right]^{T}
$$

Trim states $\overline{\mathbf{z}}^{*}$ are determined by finding a solution of the following nonlinear constrained minimization

$$
4 \text { of } 21
$$


problem for the F-16, our example aircraft in this paper:

$$
\begin{array}{cccc}
\text { minimize } & J_{\operatorname{trim}}(\mathbf{z}, \mu)=\frac{1}{2} \dot{\mathbf{z}}^{T} Q \dot{\overline{\mathbf{z}}} & & \\
\text { subject to } & h=h^{*}, & V_{T}=V_{T}^{*}, & \tan \theta=\frac{a b+\sin \psi^{*} \sqrt{a^{2}-\sin \gamma^{* 2}+b^{2}}}{a^{2}-\sin \gamma^{* 2}}, \\
& p=-\dot{\psi}^{*} \sin \theta, & q=\dot{\psi}^{*} \cos \theta \sin \phi, & r=\dot{\psi}^{*} \cos \theta \cos \phi \\
\left|\mu_{t}\right| \leq 1 & \left|\mu_{e}\right| \leq 25 & \left|\mu_{a}\right| \leq 21.5 \\
& \left|\mu_{r}\right| \leq 30 & &
\end{array}
$$

where $\gamma^{*}$ is the trimmed flight path angle satisfying $V_{T}^{*} \sin \gamma^{*}=\dot{h}^{*}, a=\cos \alpha \cos \beta$, and $b=\sin \phi \sin \beta+$ $\cos \phi \sin \alpha \cos \beta$. A numerical optimization algorithm is used to determine trim states $\mathbf{z}^{*}$ defined by $\overline{\mathbf{z}}^{*}$ because this problem cannot be solved analytically. ${ }^{3,4}$ The inequality constraints represent actuator saturation with throttle scaled in the range $[0,1]$ and with control surface deflections measured in degrees. The flight envelope is then defined as the set of feasible trimmed flight conditions $\left(\begin{array}{cccc}h^{*}, & V_{T}^{*}, \dot{h}^{*} & \dot{\psi}^{*}\end{array}\right)$ where a trimmed flight condition is feasible if there exists a valid trim state $\mathbf{z}^{*}$ and actuation vector $\mu^{*}$ for this flight condition. The stability and controllability of the feasible trimmed flight conditions are considered within a small neighborhood of each trim state. A nonlinear aircraft system 3 can be approximated by a linearization of its dynamics about a feasible trim state in a small neighborhood of the trim state. The Jacobian matrices at the trim state are obtained by using the linear perturbation model about the trim state for the linearization. Therefore, the stability and the controllability of the feasible trim state can be approximated by eigenvalues of the corresponding linear perturbation model and the controllability matrix, respectively. ${ }^{3,4}$ An aircraft trim database representing the flight envelope is then generated by characterizing each trim state in four-dimensional space $\left(h^{*}, V_{T}^{*}, \dot{h}^{*} \dot{\psi}^{*}\right)$, i.e. categorizing feasible, stable, and controllable trim states. Emergency real-time landing trajectories are generated by the Adaptive Flight Planner by using the stabilizable trim states in the damaged flight envelope. Figure 2 shows a trim database slice of the F-16 aircraft at a fixed altitude of $2000 \mathrm{ft}$ for a $20^{\circ}$ rudder jam (a) and a $5^{\circ}$ aileron jam (b). As shown in Figure 2, a green asterisk represents a naturally stable trim state while a blue asterisk implies an unstable but stabilizable trim state. The infeasible or uncontrollable trim states are unmarked.

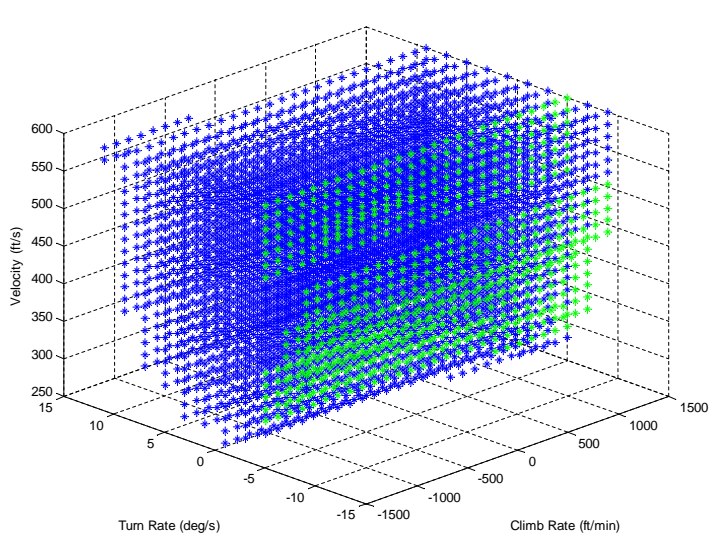

(a) $20^{\circ}$ Rudder Jam at $2000 \mathrm{ft}$

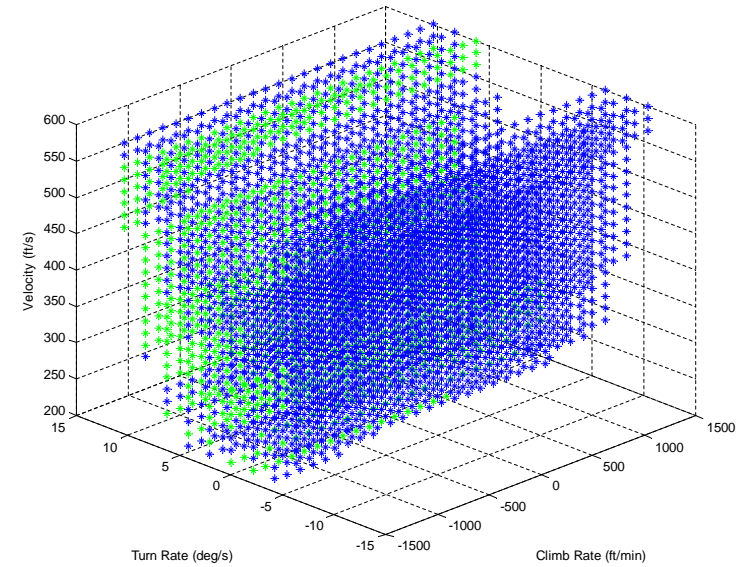

(b) $5^{\circ}$ Aileron Jam at $2000 \mathrm{ft}$

Figure 2. Trim States of the Damaged F-16 Aircraft

\section{Trim State Discovery}

In the presence of failures and/or damage, the Adaptive Flight Planner (AFP) generates emergency landing trajectories using a trim state set known to be stable or stabilizable. For failure cases that can be predicted a priori, the set of feasible post-failure trim states can be recalled from a database. For other cases, such as structural damage, offline trim state identification will not be possible. If no existing trim database is suitable for the given failure, the flight envelope, or a subset of this envelope, must be discovered online. 
Given a nonlinear dynamic system already stabilized in one feasible trim state, extrapolation of the local properties translates to determining the region of attraction around the current trim state. Researchers have studied this problem using Lyapunov function methods, ${ }^{9,10}$ with growing interest in Linear Matrix Inequality (LMI) theory to deal with nonconvex distance problems in attraction region estimation. ${ }^{11}$ Neither region of attraction nor local linearizations extrapolated away from the current state are sufficient to define the full flight envelope from the current trim state, however. Instead, the aircraft must be guided through the envelope, with further extension or contraction of the envelope at each step based on local estimate updates.

In previous work, ${ }^{6}$ we used an artificial potential field algorithm adapted from the robot motion planning community for the process of trim state discovery. A path is constructed in "trim-state-space", defined as three-dimensional coordinates $\left[\begin{array}{lll}V_{T} & \dot{h} & \dot{\psi}\end{array}\right]^{T}$, not physical space, to safely guide the aircraft through the flight envelope discovery process. This path is translated to three-dimensional physical space and altered as needed to ensure altitude and airspace constraints are satisfied. For Trim State Discovery (TSD), we assume the properties of trim states within the local neighborhood of the current trim state can be obtained, and we define a step size expected to meet region of attraction constraints. From our previous experience with damage and failure models, each identified trim state remained feasible at and below the maximum altitude where this trim state can be feasible. Therefore, we presume the trim states achievable at high altitudes are also achievable at lower altitudes, enabling the system to explore its envelope well above the terrain.

Motion planning can be categorized as static or dynamic. ${ }^{12}$ A path planner in static motion planning knows all obstacle information prior to planning, but the dynamic path planner knows obstacle information only through real-time sensing of its local environment. Researchers have studied dynamic methods of robot motion planning with obstacle avoidance using methods such as Bug algorithms (Bug 1 \& Bug 2) ${ }^{13,14}$ or Artificial Potential Fields (APF). ${ }^{15,16}$ When envelope constraint information cannot be obtained otherwise, the discovery process is activated. This implies that the path planner in TSD does not have prior information of obstacles, i.e. envelope constraints. To minimize computational overhead and handle dynamically-updated constraints we adopted the APF method for TSD. ${ }^{6}$

Without loss of generality, we assume the aircraft establishes and maintains an initial trim state just after failures and/or damage occurs, although we make no assumptions beyond stabilizability on the properties of this trim state. In the presence of failures and/or damage, the pilot or flight management system must adapt the flight plan to enable a safe landing. As the failure characteristics are initially unknown, a nominal landing trajectory may not be dynamically-feasible. TSD enables the pilot/autopilot to understand which states are stabilizable, thereby defining a flight plan that is feasible given the failure/damage effects. Given the existence of unknown, nonlinear aircraft dynamics, the stability and the controllability of a point in this envelope, a trim state, cannot be determined until the damaged aircraft transitions to the local neighborhood of that state. In previous work, ${ }^{6}$ Trim State Discovery (TSD) is defined as:

Definition Given an initial stable damaged aircraft "position" in trim-state space $\mathbf{z}_{\mathcal{T}_{0}}=\left[\begin{array}{lll}V_{T_{0}} & \dot{\psi}_{0} & \gamma_{0}\end{array}\right]^{T}$ and an ideal final approach trim state $\mathbf{z}_{\mathcal{T}_{\text {app }}}$, Trim State Discovery (TSD) is to generate a continuous path $\mathbb{T}$ in trim-state space from $\mathbf{z}_{\mathcal{T}_{0}}$ to $\mathbf{z}_{\mathcal{T}_{\text {app }}}$ where $\mathbb{T}$ is a sequence of continuous trim states and transitions. All trim states in $\mathbb{T}$ must be stabilizable in the presence of disturbances.

In our previous work, ${ }^{6}$ both two dimensional and three dimensional TSD search spaces are considered. 2D TSD searches through flight path angle $\gamma$ and turn rate $\dot{\psi}$ space with the fixed true airspeed $V_{T}$, the preferred search given that airspeed transitions typically have longer settling time. However, we observed situations in which the 2D search became trapped in a local envelope regions while extension to 3D TSD with true airspeed $V_{T}$ enables a more extensive exploration, thus increased ability to identify a safe final approach state. 3D TSD operates in the space $V_{T}, \gamma$, and $\dot{\psi}$. APF path planning in this space represents what we believe to be a novel departure from the traditional search through $3 \mathrm{D}$ physical space $x, y, z$.

In TSD, every step (local transition) in trim state space consists of two phases: exploration and exploitation. Exploration strives to understand the neighborhood of the present trim state without respect to state discovery, while exploitation seeks to determine a path $\mathbb{T}$ to a neighboring trim state by using information from exploration. In the Trim State Discovery (TSD) algorithm we previously presented, ${ }^{6}$ a stable trim state $\mathbf{z}_{\mathcal{T}_{0}}$ is established and maintained. A transition or step through trim-state-space then is modeled with a time to transition from the present state to the next state, and a time to settle in the next state such that local dynamics properties can be accurately identified. For 3D TSD, total transition time, especially settling time when airspeed changes, can be nontrivial. ${ }^{6}$ TSD, although searching through trim-state-space, must 
therefore also be concerned with ensuring the aircraft's path through physical space will not risk impact into terrain or entry into airspace unacceptable for maneuvering (e.g., for low-priority UAS). At low altitude, for example, TSD must account for terrain thus will bias its search toward positive or zero flight path angles. TSD must ultimately discover trim states with zero or negative flight path angle for approach to landing, defining a "goal state" for TSD. Discovery of descending states simply must be done once sufficient altitude is obtained.

To generate a series of trim state-space transitions connecting initial and goal final approach states, a modified Artificial Potential Field (APF) was adopted to establish a path $\mathbb{T}$ in trim state space. In trim state space, obstacles represent estimated edges of the flight envelope defined by actuator saturation or stabilizability constraints. Each edge exerts a repulsive force with sufficient strength to prevent the APF planner from violating estimated envelope constraints. The ideal final approach trim state $\mathbf{z}_{\mathcal{T}_{\text {app }}}$ exerts an attractive force. Let $\vec{F}_{A}$ and $\vec{F}_{P}$ represent the attractive and repulsive forces acting on present state $\mathbf{z}_{\mathcal{T}}$, respectively. Then $\mathbf{z}_{\mathcal{T}}$ has a resultant force $\vec{F}_{R}$ that represents the vector sum of these two forces. $\mathbf{z}_{\mathcal{T}}$ subsequently moves on this artificial potential field via transition in the direction of $\vec{F}_{R} . \vec{F}_{A}, \vec{F}_{P}$, and $\vec{F}_{R}$ as given by:

$$
\begin{aligned}
\vec{F}_{A} & =F_{A_{0}}\left[1-\exp \left(\frac{\left|\mathbf{z}_{\mathcal{T}_{\text {app }}}-\mathbf{z}_{\mathcal{T}}\right|}{\left|\mathbf{z}_{\mathcal{T}_{\text {app }}}-\mathbf{z}_{\mathcal{T}_{0}}\right|}\right)\right] \frac{\mathbf{z}_{\mathcal{T}_{\text {app }}}-\mathbf{z}_{\mathcal{T}}}{\left|\mathbf{z}_{\mathcal{T}_{\text {app }}}-\mathbf{z}_{\mathcal{T}_{c}}^{i}\right|} \\
\vec{F}_{P} & =F_{P_{0}} \sum_{i=1}^{n}\left(\frac{\mathrm{V}_{O}^{i}}{\mathrm{~V}_{a}}\right) \frac{\mathbf{z}_{\mathcal{T}}-\mathbf{z}_{\mathcal{T}_{c}}^{i}}{\left|\mathbf{z}_{\mathcal{T}}-\mathbf{z}_{\mathcal{T}_{c}}^{i}\right|} \\
\vec{F}_{R} & =\vec{F}_{A}+\vec{F}_{P}
\end{aligned}
$$

where $\mathbf{z}_{\mathcal{T}_{c}}^{i}$ is a state representing the centroid of the $i$ th dynamically-discovered obstacle and is given by:

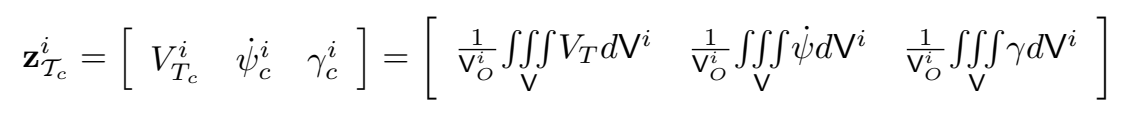

$\mathrm{V}_{O}^{i}$ and $\mathrm{V}_{a}$ are the volume of the $i$ th explored obstacle and the local attraction region, respectively. Since we define saturation boundaries but not explicit obstacle volumes, we can map centroids a certain distance from obstacle boundaries to provide appropriate repulsive field strength as envelope boundaries are approached. $F_{A_{0}}$ and $F_{P_{0}}$ are the adjustable coefficients of the attractive force and the repulsive force which also can be modified to adjust relative field strengths. TSD will follow a sequence of transitions to the final approach trim state with obstacle avoidance in the direction of a resultant force. A drawback to APF is that it can be trapped into local minima of cumulative potential. ${ }^{6}$ At the trap of local minima in the APF method, TSD switches to an edge-following method until it escapes the local minimum. In other words, $\mathbf{z}_{\mathcal{T}}$ will transition along the obstacle in the direction perpendicular to the repulsive force. Since two vectors $\vec{A}$ and $\vec{B}$ are perpendicular to the repulsive force $\vec{F}_{P}$ such that $\vec{A}$ and $\vec{B}$ are of opposite direction, direction of motion of $\mathbf{z}_{\mathcal{T}}$ is given by:

$$
\overrightarrow{\text { step }}_{i}=\left\{\begin{array}{lll}
\text { steplength } \frac{\vec{A}}{|\vec{A}|} & \text { if } & \left(\mathbf{z}_{\mathcal{T}_{i}}-\mathbf{z}_{\mathcal{T}_{i-1}}\right) \vec{A} \geq 0 \\
\text { steplength } \frac{\vec{B}}{|\vec{B}|} & \text { if } & \left(\mathbf{z}_{\mathcal{T}_{i}}-\mathbf{z}_{\mathcal{T}_{i-1}}\right) \vec{A}<0
\end{array}\right.
$$

In the edge-following method, the transition of $\mathbf{z}_{\mathcal{T}}$ depends on the distance between the local edge of the flight envelope and $\mathbf{z}_{\mathcal{T}}$. If this distance is too far, $\mathbf{z}_{\mathcal{T}}$ may diverge away from the obstacle and may return to the orginal local minimum because an attractive potential field is a function of distance from $\mathbf{z}_{\mathcal{T}_{\text {app }}}$ to $\mathbf{z}_{\mathcal{T}}$. Then $\mathbf{z}_{\mathcal{T}}$ repeats the same exploration steps and again is trapped. If $\mathbf{z}_{\mathcal{T}}$ is too close to the edge of the flight envelope, the discovered trim states may lose robustness to disturbances. Therefore, we introduce an additional repulsive force as follows:

$$
\overrightarrow{\operatorname{step}}_{i}=\left\{\begin{array}{ccc}
\overrightarrow{\operatorname{step}_{i}}+0.2 \vec{F}_{P} & \text { if } & \frac{\mathrm{V}_{O}^{i}}{\mathrm{~V}_{e}}>\epsilon_{1} \\
\overrightarrow{\mathrm{step}_{i}}-0.2 \vec{F}_{P} & \text { if } & \frac{\mathrm{V}_{O}^{o}}{\mathrm{~V}_{e}}<\epsilon_{2} \\
\overrightarrow{\mathrm{step}_{i}} & \text { if } & \epsilon_{2} \leq \frac{\mathrm{V}_{O}^{i}}{\mathrm{~V}_{e}} \leq \epsilon_{1}
\end{array}\right.
$$

where $\epsilon_{1}$ and $\epsilon_{2}$ are the prespecified coefficients representing proximity between $\mathbf{z}_{\mathcal{T}}$ and the $i$ th obstacle. 
Figure 3 shows two examples of TSD from different initial conditions, both at initial altitude $10000 \mathrm{ft}$ MSL. As shown, the AFP-generated path progresses to a final approach goal state followed by a termination sequence in which local exploration around the final approach state is conducted to ensure the final approach and neighboring states are well-understood. Our damaged F-16 model with aileron jammed at -10 degrees was used in these three dimensional TSD simulations, as previously described in Yi and Atkins. ${ }^{6}$ The final trim state is specified as $\mathbf{z}_{\mathcal{T}_{\text {app }}}=\left[\begin{array}{ccc}V_{T} & 0^{\circ} & -3^{\circ}\end{array}\right]^{T}$, with airspeed $V_{T}$ determined during exploration. The initial trim state is defined as $\mathbf{z}_{\mathcal{T}_{0}}=\left[\begin{array}{lll}400 \mathrm{ft} / \mathrm{s} & 15.5^{\circ} & 2.9^{\circ}\end{array}\right]^{T}$ for the first simulation (left) and $\mathbf{z}_{\mathcal{T}_{0}}=\left[\begin{array}{lll}450 \mathrm{ft} / \mathrm{s} & -15^{\circ} & -1.3^{\circ}\end{array}\right]^{T}$ for the second simulation (right). The green dots indicate stablizable trim states, and blue dots and line represent the TSD path. As shown, despite the non-convex flight envelope, more challenging to traverse than typical convex nominal envelope geometries, the AFP-based TSD algorithm was able to identify a near-direct path from initial to a valid final approach state.

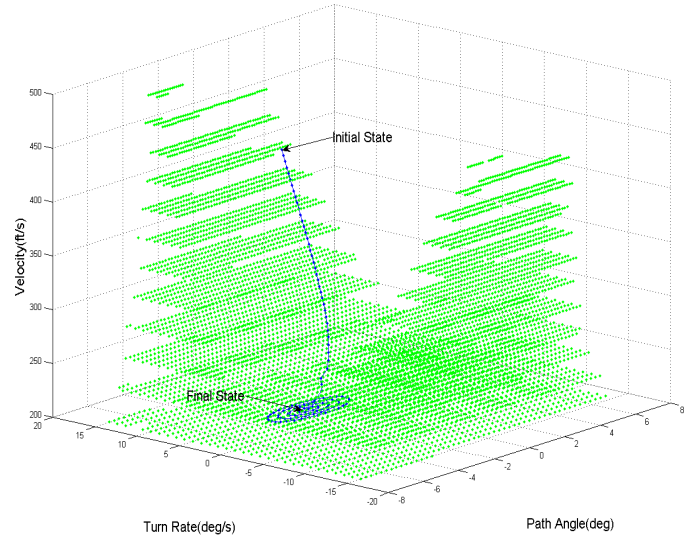

(a) $V_{T}=400 \mathrm{ft} / \mathrm{s}, \dot{\psi}=15.5^{\circ}$, and $\gamma=2.9^{\circ}$

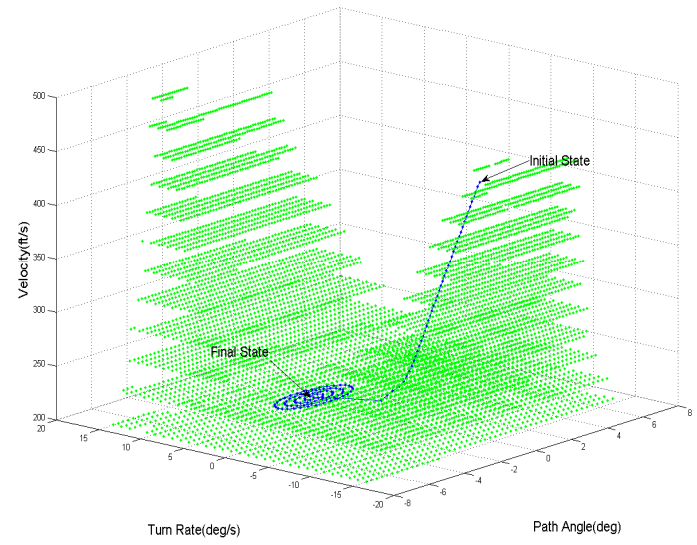

(b) $V_{T}=450 \mathrm{ft} / \mathrm{s}, \dot{\psi}=-16^{\circ}$, and $\gamma=-1.3^{\circ}$

Figure 3. 3D Trim State Discovery Examples for Different Initial Trim States

\section{Turning Dubins Vehicle Trajectory}

Using trim states from the trim database or from Trim State Discovery, the Adaptive Flight Planner (AFP) builds a real-time emergency landing trajectory to guide the damaged aircraft to a chosen landing runway. If the aircraft can fly straight after failures and/or damage, a Dubins path is generated by the AFP. Severe damage cases, however, such as a previously-studied left wing structural damage case ${ }^{4}$ may render the aircraft unable to maintain a straight flight path. In previous work, ${ }^{5}$ we defined the concept of a Turning Dubins Vehicle as an extension of the Dubins path landing solution as follows:

Definition (Turning Dubins Vehicle (TDV)) A Turning Dubins Vehicle is a planar vehicle that is constrained to move along paths of curvature bounded both above and below, without reversing direction and maintaining a constant speed.

Let $\sigma:[0, T] \rightarrow \mathbb{R}^{2}$ be a curve for the TDV that is twice differentiable for maneuver times $T \geq 0$, and $\mathcal{C}(s)$ represent a natural representation of $\sigma$ with respect to $c_{i}$. Since the magnitude of the curvature of $\sigma$ is bounded above by the reciprocal of the minimum turning radius $r_{m}$ and below by the reciprocal of the maximum turning radius $r_{M}$, let $\Sigma$ represent the set of possible curves for the TDV, i.e., $\Sigma=$ $\left\{\sigma \mid k \in\left[\frac{1}{r_{M}}, \frac{1}{r_{m}}\right]\right\}$ where $\vec{k}$ is the curvature vector of $\sigma$, and let $\Sigma_{c}$ be the set of circular curves for the TDV as follows:

$$
\Sigma_{c}=\left\{\mathcal{O}\left(\vec{r}_{c}, r, \operatorname{sgn}(\dot{\psi})\right) \mid \quad r_{m} \leq r \leq r_{M}, \quad \operatorname{sgn}(\dot{\psi})=\left\{\begin{array}{ll}
+1 & \text { if } \dot{\psi}>0 \\
-1 & \text { if } \dot{\psi}<0
\end{array}\right\}\right.
$$


where $\mathcal{O}\left(\vec{r}_{c}, r, \operatorname{sgn}(\dot{\psi})\right):\left[0, T_{\mathcal{O}}\right] \rightarrow \mathbb{R}^{2}$ represents a circle of radius $r$ with center $\vec{r}_{c}$ and direction of motion $\operatorname{sgn}(\dot{\psi})$ where $T_{\mathcal{O}}$ denotes the maneuver time during $\mathcal{O}$. For a given center $\vec{r}_{c}$ in $\mathbb{R}^{2}$ and two given points $\vec{r}_{p_{i}}$ and $\vec{r}_{p_{f}}$ in $\mathbb{R}^{2}$, let $a\left(\vec{r}_{c}, \vec{r}_{p_{i}}, \vec{r}_{p_{f}}\right):\left[0, T_{a}\right] \rightarrow \mathbb{R}^{2}$ be a circular arc connecting $\vec{r}_{p_{i}}$ and $\vec{r}_{p_{f}}$ with arc center $\vec{r}_{c}$ and let $\mathcal{A}=\left\{a\left(\vec{r}_{c}, \vec{r}_{p_{i}}, \vec{r}_{p_{f}}\right) \mid \quad \vec{r}_{c}, \vec{r}_{p_{i}}, \vec{r}_{p_{f}} \in \mathbb{R}^{2}\right\}$

To obtain the landing trajectory, we identify a reference arc that can be followed by alternating segments of two different turning radii that include a predefined safety factor sufficient for disturbance rejection. Since the perpendicular bisector of a chord passes through the center of the circle, ${ }^{17}$ let $\mathcal{A}_{r}$ be the set of possible reference arcs connecting centers

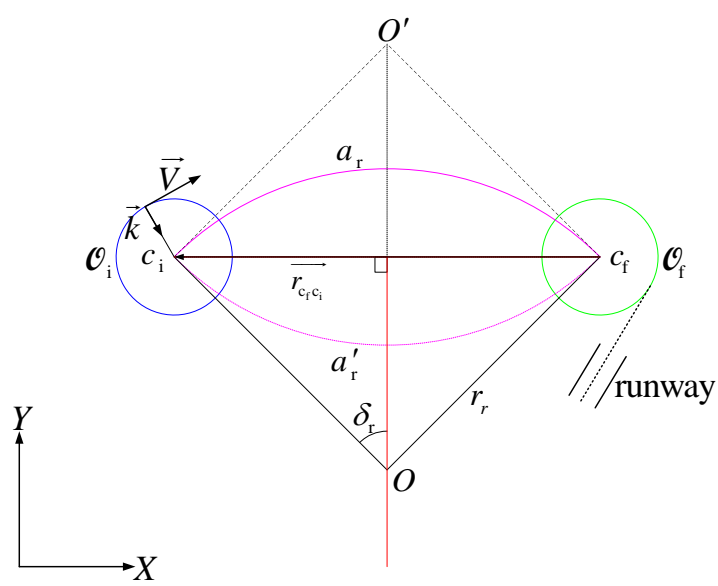

Figure 4. Reference Arcs $c_{i}$ and $c_{f}$ as follows :

$$
\mathcal{A}_{r}=\left\{a\left(\vec{r}_{O}, \vec{r}_{c_{i}}, \vec{r}_{c_{f}}\right) \mid \quad \vec{r}_{O}=\frac{1}{2}\left(\vec{r}_{c_{i}}+\vec{r}_{c_{f}}\right)+\lambda\left(\cos \zeta_{O} \hat{I}+\sin \zeta_{O} \hat{J}\right), \quad \lambda \in \mathbb{R}\right\}
$$

where $\vec{r}_{O}$ is a vector to the center $O$ of the reference arc, and $\zeta_{O}=\arctan \left(-\frac{x_{c_{f} c_{i}}}{y_{c_{f} c_{i}}}\right)$ given $\vec{r}_{c_{f} c_{i}}=x_{c_{f} c_{i}} \hat{I}+$ $y_{c_{f} c_{i}} \hat{J}$. Note that there also exists a dual reference $\operatorname{arc} a_{r}^{\prime} \in \mathcal{A}_{r}$ because the direction of the unit vector represeting the perpendicular bisector can be reversed, as shown in Figure 4. Using $\vec{r}_{O c_{i}}$, the radius $r_{r}$, angle $\delta_{r}$, and length $l_{r}$ of the reference arc $a_{r} \in \mathcal{A}_{r}$, as shown in Figure 4, are expressed as $\left\|\vec{r}_{O c_{i}}\right\|$, $\arccos \left(\frac{\lambda}{r_{r}}\right)$, and $2 r_{r} \delta_{r}$, respectively. When we determine alternating segments of two different turning radii, the below definition of a product of two arcs is important. If $a_{2 i-1}\left(T_{2 i-1}-T_{2 i-2}\right)=a_{2 i}(0)$ where $a_{2 i-1}$ and $a_{2 i}$ are the arcs of $\mathcal{O} \in \Sigma_{c}$ intercepted by $a_{r} \in \mathcal{A}_{r}$, then we define a product of two arcs as :

$$
b_{i}=a_{2 i-1} * a_{2 i}= \begin{cases}a_{2 i-1}\left(t-T_{2 i-2}\right), & T_{2 i-2} \leq t \leq T_{2 i-1} \\ a_{2 i}\left(t-T_{2 i-1}\right), & T_{2 i-1} \leq t \leq T_{2 i}\end{cases}
$$

where $T_{0}=0$. The following theorem and corollary that we have proven previously ${ }^{5}$ present how to arrange a sequence of two different turning radii and a sequence of alternate turning arcs for the TDV.

Theorem IV.1 Let $a_{r} \in \mathcal{A}_{r}$, and let $\mathcal{O}_{1}\left(c_{1}, r_{1}\right.$, sgn $\left.(\dot{\psi})\right)$ and $\mathcal{O}_{2}\left(c_{2}, r_{2}, \operatorname{sgn}(\dot{\psi})\right)$ be in $\Sigma_{c}$ with $r_{1} \neq r_{2}$. Let $a_{1}$ be the intercepted arc of $\mathcal{O}_{1}$ with $a_{r}$ and center $c_{1}$ such that $a_{1}\left(c_{1}, p_{0}, p_{1}\right):\left[0, T_{1}\right] \rightarrow \mathbb{R}^{2}$ where $p_{0}$ and $p_{1}$ lie on $a_{r}$. Let $a_{2}$ be the intercepted arc of $\mathcal{O}_{2}$ with $a_{r}$ and center $c_{2}$ such that $a_{2}\left(c_{2}, p_{1}, p_{2}\right):\left[0, T_{2}-T_{1}\right] \rightarrow \mathbb{R}^{2}$ where $p_{2}$ lies on $a_{r}$. Suppose $c_{1}$ lies on arc $a_{r}$. If $c_{2}$ is located on ray $\overrightarrow{p_{1} c_{1}}$ with distance $r_{2}$ from $p_{1}$, then $\mathcal{O}_{1}$ and $\mathcal{O}_{2}$ are tangent at $p_{1}$. Moreover, the change in heading angle of the TDV over $a_{1} * a_{2}$ is $2 \pi-\left|4 \delta_{1}-2 \delta_{2}\right|$ if $\operatorname{sgn}(\dot{\psi})>0$ and $2 \pi+\left|4 \delta_{1}-2 \delta_{2}\right|$ if $\operatorname{sgn}(\dot{\psi})<0$ where $\delta_{1}=\arcsin \left(\frac{r_{1}}{2 r_{r}}\right)$ and $\delta_{2}=\arccos \left(\frac{\vec{a}_{O c_{2}} \cdot \vec{a}_{O p_{1}}}{\left\|\vec{a}_{O c_{2}}\right\| r_{r}}\right)$.

Note that $2 \delta_{1}>\delta_{2}$ if $r_{1}>r_{2}$, and $2 \delta_{1}<\delta_{2}$ if $r_{1}<r_{2}$.

Corollary IV.2 Let $a_{r} \in \mathcal{A}_{r}$. Let $\mathcal{O}_{2 i-1}\left(c_{2 i-1}, r_{1}, \operatorname{sgn}(\dot{\psi})\right)$ and $\mathcal{O}_{2 i}\left(c_{2 i}, r_{2}, \operatorname{sgn}(\dot{\psi})\right)$ be in $\Sigma_{c}$ with $r_{1} \neq r_{2}$ for all $i \in \mathbb{N}$. Let $a_{2 i-1}$ be the intercepted arc of $\mathcal{O}_{2 i-1}$ with $a_{r}$ and center $c_{2 i-1}$ such that $a_{2 i-1}\left(c_{2 i-1}, p_{2 i-2}, p_{2 i-1}\right):\left[0, T_{2 i-1}-T_{2 i-2}\right] \rightarrow \mathbb{R}^{2}$. Suppose $c_{1}, p_{0}$, and $p_{1}$ lie on $a_{r}$. Let $a_{2 i}$ be the intercepted arc of $\mathcal{O}_{2 i}$ with $a_{r}$ and center $c_{2 i}$ such that $a_{2 i}\left(c_{2 i}, p_{2 i-1}, p_{2 i}\right):\left[0, T_{2 i}-T_{2 i-1}\right] \rightarrow \mathbb{R}^{2}$. If $c_{2 i}$ is located on ray $\overrightarrow{p_{2 i-1} c_{2 i-1}}$ with distance $r_{2}$ from $p_{2 i-1}$ and $c_{2 i+1}$ is located on ray $\overrightarrow{p_{2 i} c_{2 i}}$ with distance $r_{1}$ from $p_{2 i}$ for all $i \in \mathbb{N}$ where $p_{2 i-1}$ and $p_{2 i}$ lie on $a_{r}$, then $\mathcal{O}_{2 i-1}$ and $\mathcal{O}_{2 i}$ are tangent at $p_{2 i-1}$, and $\mathcal{O}_{2 i}$ and $\mathcal{O}_{2(i+1)-1}$ at $p_{2 i}$ tangent such that $c_{2 i-1}$ and $c_{2(i+1)-1}$ lie on $a_{r}$ for all $i \in \mathbb{N}$. Consider the set $\left\{b_{i} \mid b_{i}=a_{2 i-1} * a_{2 i}, i \in \mathbb{N}\right\}$. Moreover, the change in heading angle of the TDV over $b_{1} * b_{2} * \cdots * b_{i}$ is $i\left(2 \pi-\left|4 \delta_{1}-2 \delta_{2}\right|\right)$ if sgn $(\dot{\psi})>0$ and $i\left(2 \pi+\left|4 \delta_{1}-2 \delta_{2}\right|\right)$ if $\operatorname{sgn}(\dot{\psi})<0$ for all $i \in \mathbb{N}$ where $\delta_{1}=\arcsin \left(\frac{r_{1} / 2}{r_{r}}\right)$ and $\delta_{2}=\arccos \left(\frac{\vec{a}_{O c_{2}} \cdot \vec{a}_{O p_{1}}}{\|\| \vec{a}_{O c_{2}} \| r_{r}}\right)$. 
The proof of Corollary IV.2 is basically the same as the proof of Theorem IV.1 and is completed by mathmatical induction. ${ }^{5}$ In the proof of Corollary IV.2, since $\mathcal{O}_{2 i-1}$ and $\mathcal{O}_{2 i}$ are tangent at $p_{2 i-1}$ and $a_{2 i-1}\left(T_{2 i-1}-T_{2 i-2}\right)=a_{2 i}(0)=p_{2 i-1}$ for all $i \in \mathbb{N}, b_{i}$ is defined for all $i \in \mathbb{N}$. If $b_{i}\left(T_{2 i}-T_{2 i-2}\right)=b_{i+1}(0)$ where $b_{i}=a_{2 i-1} * a_{2 i}$ and $b_{i+1}=a_{2(i+1)-1} * a_{2(i+1)}$, then we define a product of two products as :

$$
b_{i} * b_{i+1}= \begin{cases}b_{i}\left(t-T_{2 i-2}\right), & T_{2 i-2} \leq t \leq T_{2 i} \\ b_{i+1}\left(t-T_{2 i}\right), & T_{2 i} \leq t \leq T_{2 i+2}\end{cases}
$$

where $T_{0}=0$. Since $\mathcal{O}_{2 i}$ and $\mathcal{O}_{2(i+1)-1}$ are tangent at $p_{2 i}$ and $a_{2 i}\left(T_{2 i}-T_{2 i-1}\right)=a_{2 i+1}(0)=p_{2 i}$ for all $i \in \mathbb{N}, b_{1} * b_{2} * \cdots * b_{i}$ is defined for all $i \in \mathbb{N}$.

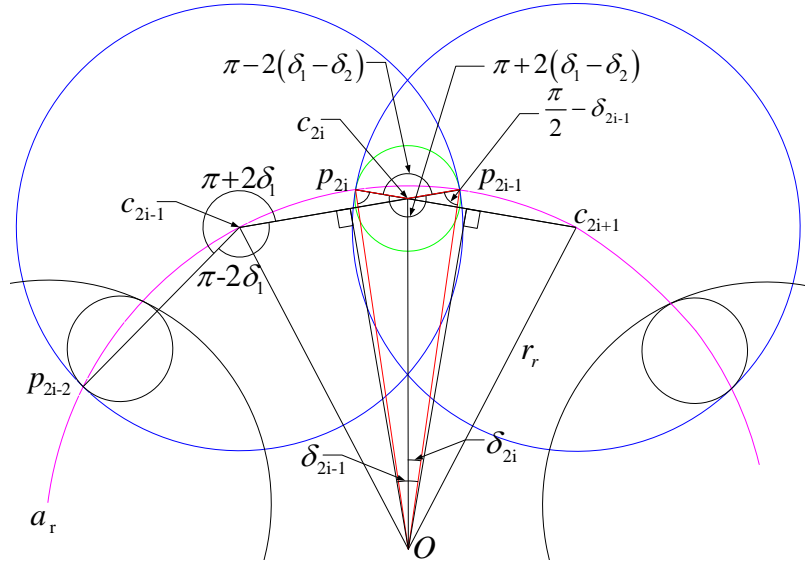

(a) Product of $n$ Arcs if $r_{1}>r_{2}$

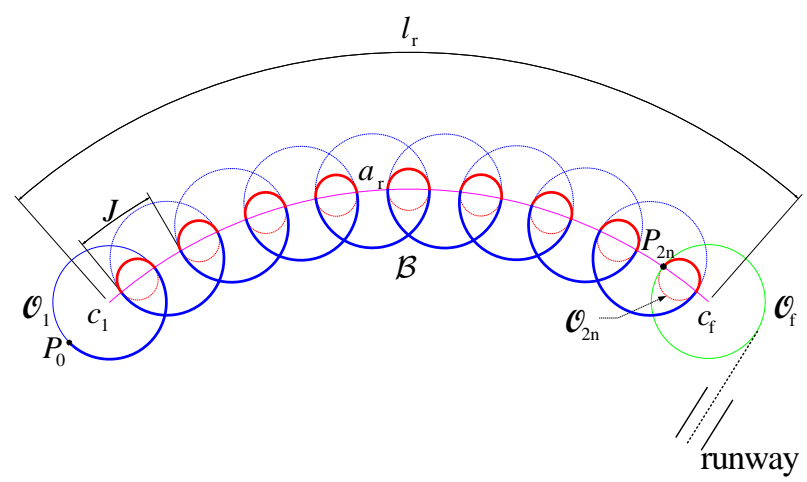

(c) Feasibility Condition if $r_{1}>r_{2}$ and $\operatorname{sgn}(\dot{\psi})>0$

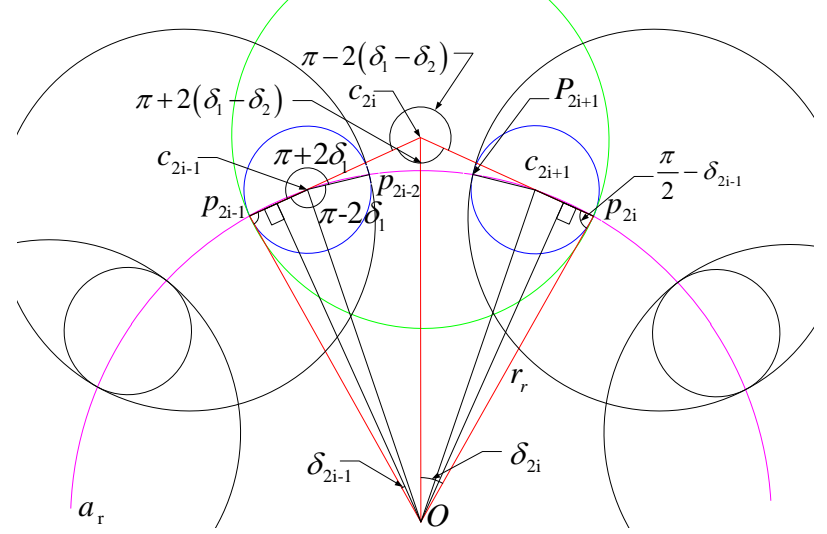

(b) Product of $n$ Arcs if $r_{1}<r_{2}$

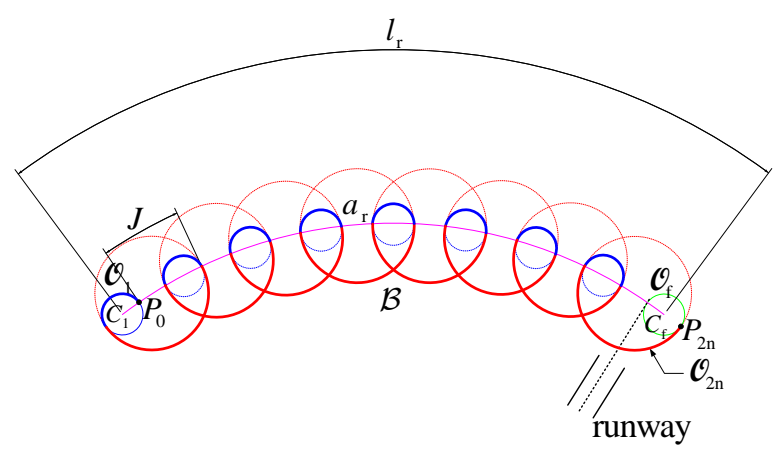

(d) Feasibility Condition if $r_{1}<r_{2}$ and $\operatorname{sgn}(\dot{\psi})>0$

Figure 5. Product of $n$ Arcs and Feasibility Condition

Let $\left\{b_{i} \mid b_{i}=a_{2 i-1} * a_{2 i}, \quad i \in \mathbb{N}\right\}$ represent the set of products of alternate turning arcs for the TDV in $a_{r} \in \mathcal{A}_{r}$ such that Corollary IV.2 holds, denoted by $\mathcal{B}$. Although $\mathcal{B}$ follows a reference $\operatorname{arc} a_{r}$ from the previous corollary, the final circular curve $\mathcal{O}_{f}$ and the $2 n$th circular curve $\mathcal{O}_{2 n}$ for the TDV are not guaranteed tangent at $p_{2 n}$ after $n$ sequences. The following theorem that we have proven previously ${ }^{5}$ describes the horizontal feasibility condition about $a_{r}$ for the TDV to reach the selected runway.

Theorem IV.3 Let $a_{r} \in \mathcal{A}_{r}$, and let $n \in \mathbb{N}$ be given. Suppose $\mathcal{B}=\left\{b_{i} \mid b_{i}=a_{2 i-1} * a_{2 i}, i \in\{1,2, \cdots, n\}\right\}$ over $a_{r}$ such that Corollary IV.2 holds. Let $\mathcal{O}_{1}\left(c_{i}, r_{1}, \operatorname{sgn}(\dot{\psi})\right)$ and $\mathcal{O}_{f}\left(c_{f}, r_{1}, \operatorname{sgn}(\dot{\psi})\right)$ represent the initial and final circular curves, respectively. Then there exists an $a_{r}$ such that the final position in $b_{n} \in \mathcal{B}, \vec{r}_{p_{2 n}}$, lies on an intersection point of $\mathcal{O}_{f}$ and $a_{r}$ if and only if $r_{r}$ satisfies the horizontal feasibility condition:

$$
2 \delta_{r}=n\left|4 \delta_{1}-2 \delta_{2}\right|
$$


where $\delta_{r}=\arccos \left(\frac{\lambda}{r_{r}}\right), \delta_{1}=\arcsin \left(\frac{r_{1}}{2 r_{r}}\right)$, and $\delta_{2}=\arctan \left(\frac{\sqrt{r_{r}^{2}-\frac{r_{1}^{2}}{4}}}{\frac{r_{r}^{2}}{r_{2}}-\frac{r_{1}}{2}}\right)$. Therefore, the TDV can reach the selected runway.

The essence of the proof of Theorem IV.3 is to show that a center of an additional circular curve $\mathcal{O}_{2 n+1}$ from Corollary IV.2 is a center of the final circular curve $\mathcal{O}_{f}$.

Let $a_{r} \in \mathcal{A}_{r}$, and let $l_{r}$ represent the length of $a_{r}$ from the center $c_{1}$ to the center $c_{f}$ from the definition of $\mathcal{A}_{r}$. Let $\mathcal{J}$ represent the lenght of $a_{r}$ traversed over $b \in \mathcal{B}$ in $a_{r}$. From Corollary IV.2, $\mathcal{J}$ is independent of the index $i$ in $\mathcal{B}$. Multiplying Eq. 18 by the radius $r_{r}$ of the reference arc, we obtain the horizontal feasibility condition about the length of $a_{r}$ :

$$
l_{r}=n \mathcal{J}
$$

where $\mathcal{J}=\left|2 r_{1}-2 r_{2}\right|$ if $a_{r}$ is a straight line, and $\mathcal{J}=\left|4 r_{r} \delta_{1}-2 r_{r} \delta_{2}\right|$ otherwise from Theorem IV.1. Thus, $l_{r}$ must be an integer multiple of $\mathcal{J}$ for the runway to be reached via $b_{1} * b_{2} * \cdots * b_{i}$ where $b_{i} \in \mathcal{B}$ for all $i \in\{1,2, \cdots, n\}$. Since $a_{r} \in \mathcal{A}_{r}$ evolves from a straight line to a circle as $\lambda$ in Eq. 15 is varied from $\infty$ to $-\infty$, we can find a lower bound for $n$ in $\mathcal{B}=\left\{b_{i} \mid b_{i}=a_{2 i-1} * a_{2 i}, \quad i \in\{1,2, \cdots, n\}\right\}$ over $a_{r}$ representing a straight line. Moreover, since the radius of TDV's curvature is in $\left[r_{m}, r_{M}\right], \mathcal{J}$ is bounded. Let $n_{m}$ represent the minimum value of $n$ in $\mathcal{B}$ over $a_{r}$ satisfying the horizontal feasibility condition such that $\mathcal{J}$ has the maximum value, and let $a_{r_{m}}$ represent the reference arc having $n_{m}$. In the next theorem, we consider the minimum sequence of alternating extreme turning arcs satisfying the horizontal feasibility condition 18.

Theorem IV.4 Let $a_{r} \in \mathcal{A}_{r}$, and let $n \in \mathbb{N}$ be given. Suppose $\mathcal{B}=\left\{b_{i} \mid b_{i}=a_{2 i-1} * a_{2 i}, i \in\{1,2, \cdots, n\}\right\}$ over $a_{r}$ such that Corollary IV.2 holds. Let $\mathcal{O}_{1}=\left(c_{i}, r_{1}, \operatorname{sgn}(\dot{\psi})\right)$ and $\mathcal{O}_{f}=\left(c_{f}, r_{1}, \operatorname{sgn}(\dot{\psi})\right)$ represent the initial and final circular curves, respectively. Suppose $a_{r}$ satisfies the horizontal feasibility condition 19. Then $n_{m}=\left\lceil\frac{\left\|\vec{r}_{c_{f} c_{i}}\right\|}{\left|2 r_{1}-2 r_{2}\right|}\right\rceil$ and there exists $a_{r_{m}}$ such that $\mathcal{O}_{2 n}$ and $\mathcal{O}_{f}$ are tangent at $p_{2 n}$ where

$$
\begin{array}{lllll}
r_{1}=r_{M} & \text { and } & r_{2}=r_{m} & \text { if } & r_{1}>r_{2} \\
r_{1}=r_{m} & \text { and } & r_{2}=r_{M} & \text { if } & r_{1}<r_{2}
\end{array}
$$

The following lemma to bound the maximum value of $J$ is used to prove the above theorem. ${ }^{5}$

Lemma IV.5 If circular curves of two distinct radii satisfy the condition:

$$
\begin{array}{lllll}
r_{1}=r_{M} & \text { and } & r_{2}=r_{m} & \text { if } & r_{1}>r_{2} \\
r_{1}=r_{m} & \text { and } & r_{2}=r_{M} & \text { if } & r_{1}<r_{2}
\end{array}
$$

then $J$ has the maximum value for all $a_{r} \in \mathcal{A}_{r}$.

To prove the above lemma, we use other expressions of $\delta_{2}$ given by:

$$
\delta_{2}=\left\{\begin{array}{l}
\delta_{1}-\arctan \left(\frac{\frac{r_{1}}{2}-r_{2}}{\sqrt{r_{r}^{2}-\frac{r_{1}^{2}}{4}}}\right) \quad \text { if } \quad r_{1}>r_{2} \\
\delta_{1}+\arctan \left(\frac{r_{2}-\frac{r_{1}}{2}}{\sqrt{r_{r}^{2}-\frac{r_{1}^{2}}{4}}}\right) \text { if } \quad r_{1}<r_{2}
\end{array}\right.
$$

The essence of the proof of Theorem IV.4 is to show that the number $n$ of products of alternating turning arcs is greater than or equal to the length of $a_{r}$ representing a straight line divided by the maximum of the length of $\mathcal{J}$. Note that $r_{r}$ of $a_{r} \in \mathcal{A}_{r}$ has the following constraints:

$$
r_{r}>\frac{r_{1}}{2}, \quad \text { and } \quad r_{r} \geq \frac{\left\|\vec{r}_{c_{f} c_{i}}\right\|}{2}
$$




\section{3D Turning Dubins Vehicle Trajectory}

In the previous analysis of the Turning Dubins Vehicle, we presume the longitudinal and lateral aircraft dynamics are fully-decoupled, and that we can achieve a flight path angle that yields the necessary altitude change from the initial state to the final (landing) state. We now relax this assumption to consider constraints on flight path angle as a function of turning radius. For simplicity, we assume a flight path angle $\gamma$ is constant throughout descending flight to landing. Although the possible range of $\gamma$ will vary as a function of turn radius, we define $\gamma_{m}$ and $\gamma_{M}$ as the minimum and maximum flight path angles of the TDV at the initial altitude, respectively, achievable for all turn radii in the range $\left[r_{m}, r_{M}\right]$. Given the initial position $\vec{r}_{p_{I}}$ of the TDV and the position $\vec{r}_{p_{f}}$ of the landing runway, let $h_{0}$ be the initial altitude of the TDV. Let $r_{m}$ and $r_{M}$ be given. By Corollary IV.2, the change in heading angle of the TDV in $\mathcal{B}=\left\{b_{i} \mid b_{i}=a_{2 i-1} * a_{2 i}, \quad i \in\{1,2, \cdots, n\}\right\}$ for all $n \in \mathbb{N}$ is given by:

$$
\begin{array}{llc}
n\left(2 \pi-\left|4 \delta_{1}-2 \delta_{2}\right|\right) & \text { if } & \operatorname{sgn}(\dot{\psi})>0 \\
n\left(2 \pi+\left|4 \delta_{1}-2 \delta_{2}\right|\right) & \text { if } & \operatorname{sgn}(\dot{\psi})<0
\end{array}
$$

where $\delta_{1}=\arcsin \left(\frac{r_{1}}{2 r_{r}}\right)$, and $\delta_{2}=\delta_{1}-\arctan \left(\frac{\frac{r_{1}}{2}-r_{2}}{\sqrt{r_{r}^{2}-\frac{r_{1}^{2}}{4}}}\right)$ if $r_{1}>r_{2}$ and $\delta_{2}=\delta_{1}+\arctan \left(\frac{r_{2}-\frac{r_{1}}{2}}{\sqrt{r_{r}^{2}-\frac{r_{1}^{2}}{4}}}\right)$ if $r_{1}<r_{2}$.

The minimum value $n_{m}$ of $n$ in $\mathcal{B}$ over $a_{r}$ is determined by Theorem IV.4. Let $s_{\mathcal{B}}$ represent a natural parameter of $\sigma$ when the TDV finishes $\mathcal{B}=\left\{b_{i} \mid b_{i}=a_{2 i-1} * a_{2 i}, \quad i \in\{1,2, \cdots, n\}\right\}$ over $a_{r}$ satisfying the feasibility condition 18 . Given $\vec{r}_{p_{0}}, \vec{r}_{p_{f}}, r_{m}$, and $r_{M}$, the length $s_{\mathcal{B}}$ of $\mathcal{B}$ is then given by:

$$
s_{\mathcal{B}}=\left\{\begin{array}{llc}
n \pi\left(r_{1}+r_{2}\right)-2 n \delta_{1}\left|r_{1}-r_{2}\right|-2 \delta_{r} r_{2} & \text { if } & \operatorname{sgn}(\dot{\psi})>0 \\
n \pi\left(r_{1}+r_{2}\right)+2 n \delta_{1}\left|r_{1}-r_{2}\right|+2 \delta_{r} r_{2} & \text { if } & \operatorname{sgn}(\dot{\psi})<0
\end{array}\right.
$$

for all $n \geq n_{m}$ using feasibility condition 18 and Eq. 24. However, we don't consider the arc length from the initial position $\vec{r}_{p_{I}}$ of the TDV to the position $\vec{r}_{p_{0}}$ in the first circular arc $a_{1}$ and the arc length from $\vec{r}_{p_{2} n}$ in $b_{n} \in \mathcal{B}$ to $\vec{r}_{p_{f}}$ in Eq. 28. Let $\psi_{I}$ be the initial heading of the TDV, and let $\psi_{1}$ be the heading in the final point $p_{1}$ of the initial circular arc $a_{1}$. Then the difference $\left|\psi_{1}-\psi_{I}\right|$ between two headings is determined using the dot product of $\vec{r}_{c_{i} p_{I}}$ and $\vec{r}_{c_{i} p_{1}}$ according to the travel direction. We also determine the difference $\left|\psi_{f}-\psi_{2} n\right|$ of the heading $\psi_{f}$ of the landing runway and the heading $\psi_{2} n$ of the final position in $b_{n} \in \mathcal{B}$ in the same manner. Therefore, we now define $s_{\mathcal{B}}$ as the length of the TDV trajectory from the initial position of the TDV to the position of the landing runway in $\mathcal{B}$ :

if $r_{1}>r_{2}$,

$s_{\mathcal{B}}=\left\{\begin{array}{lll}\pi\left[(n-1) r_{1}+n r_{2}\right]-2 \delta_{1}\left[(n-1) r_{1}-n r_{2}\right]-2 \delta_{r} r_{2}+\left(\left|\psi_{1}-\psi_{I}\right|+\left|\psi_{f}-\psi_{2 n}\right|\right) r_{1} & \text { if } & \operatorname{sgn}(\dot{\psi})>0 \\ \pi\left[(n-1) r_{1}+n r_{2}\right]+2 \delta_{1}\left[(n-1) r_{1}-n r_{2}\right]+2 \delta_{r} r_{2}+\left(\left|\psi_{1}-\psi_{I}\right|+\left|\psi_{f}-\psi_{2 n}\right|\right) r_{1} & \text { if } & \operatorname{sgn}(\dot{\psi})<0\end{array}\right.$

if $r_{1}<r_{2}$,

$s_{\mathcal{B}}=\left\{\begin{array}{lll}\pi\left[(n-1) r_{1}+n r_{2}\right]+2 \delta_{1}\left[(n-1) r_{1}-n r_{2}\right]-2 \delta_{r} r_{2}+\left(\left|\psi_{1}-\psi_{I}\right|+\left|\psi_{f}-\psi_{2 n}\right|\right) r_{1} & \text { if } & \operatorname{sgn}(\dot{\psi})>0 \\ \pi\left[(n-1) r_{1}+n r_{2}\right]-2 \delta_{1}\left[(n-1) r_{1}-n r_{2}\right]+2 \delta_{r} r_{2}+\left(\left|\psi_{1}-\psi_{I}\right|+\left|\psi_{f}-\psi_{2 n}\right|\right) r_{1} & \text { if } & \operatorname{sgn}(\dot{\psi})<0\end{array}\right.$

where $\delta_{1}=\arcsin \left(\frac{r_{1}}{2 r_{r}}\right)$, and $\delta_{2}=\delta_{1}-\arctan \left(\frac{\frac{r_{1}}{2}-r_{2}}{\sqrt{r_{r}^{2}-\frac{r_{1}^{2}}{4}}}\right)$ if $r_{1}>r_{2}$ and $\delta_{2}=\delta_{1}+\arctan \left(\frac{r_{2}-\frac{r_{1}}{2}}{\sqrt{r_{r}^{2}-\frac{r_{1}^{2}}{4}}}\right)$ if $r_{1}<r_{2}$.

In this work, we assume $\gamma_{m}$ is negative. This assumption is appropriate for the TDV to reach the selected landing runway. If the TDV satisfies the vertical feasibility condition:

$$
\gamma_{m} \leq \arctan \left(-\frac{h_{0}}{s_{\mathcal{B}}}\right) \leq \gamma_{M}
$$


then the TDV can reach the landing runway. $s_{\mathcal{B}}$ is a function of $n$ in $\mathcal{B}$ over $a_{r}$, and thus is noncontinuous on $\left\{n \in \mathbb{N} \mid n \geq n_{m}\right\}$ for the fixed $r_{1}$ and $r_{2}$. However, $s_{\mathcal{B}}$ in the condition 31 depends on the range of the turning radius as well as the initial position of the TDV and the position of the selected landing runway. Changing $r_{1}$ and/or $r_{2}$ on $\left[r_{m}, r_{M}\right], s_{\mathcal{B}}$ can be continuous for each $n \in\left\{n \in \mathbb{N} \mid n \geq n_{m}\right\}$. Without loss of generality, let $r_{1}=r_{M}$ for the case where $r_{1}>r_{2}$. From previous work, ${ }^{5}$ we know that if $r_{2}=r_{m}$, then $\mathcal{J}$ has the maximum value for all $a_{r} \in \mathcal{A}_{r}$. Therefore, $\mathcal{J}$ is decreased for all $a_{r} \in \mathcal{A}_{r}$ as $r_{2}$ is increased from $r_{m}$ to $r_{M}-\left[\frac{\left\|\vec{r}_{C f C i}\right\|}{2 n}\right]$ where $n \in\left\{n \in \mathbb{N} \mid n \geq n_{m}\right\}$. Since $l_{r}=n \mathcal{J}$, the length of $a_{r}$ from the center $c_{1}$ to the center $c_{f}$ should be decreased for $n$ to be constant. Since $a_{r} \in \mathcal{A}_{r}$ evolves from a straight line to a circle as $\lambda$ is varied from $\infty$ to $-\infty, r_{r}$ should be increased if $a_{r}$ was the minor reference arc when $r_{2}=r_{m}$, or be decreased to $\frac{\left\|\vec{r}_{C f C i}\right\|}{2}$ if $a_{r}$ was the major reference arc when $r_{2}=r_{m}$. If $r_{2}=r_{M}-\frac{\left\|\vec{r}_{C f C i}\right\|}{2 n}$, then the reference arc $a_{r}$ is a straight line because $\frac{\left\|\vec{r}_{C f C i}\right\|}{\left|2 r_{M}-2 r_{2}\right|}=n$. Furthermore, $r_{M}-\left[\frac{\left\|\vec{r}_{C f C i}\right\|}{2 n}\right]$ has the limit $r_{M}$ as $n$ approaches $\infty$. Therefore, $r_{r}$ is increased if $a_{r}$ was the minor reference arc when $r_{2}=r_{m}$, or is decreased to $\frac{\left\|\vec{r}_{C f C i}\right\|}{2}$ and is increased if $a_{r}$ was the major reference arc when $r_{2}=r_{m}$ as $r_{2}$ is increased on $\left[r_{m}, r_{M}-\frac{\left\|\vec{r}_{C f C i}\right\|}{2 n}\right]$ where $n \in\left\{n \in \mathbb{N} \mid n \geq n_{m}\right\}$. The case where $r_{1}<r_{2}$ is the same as the case when $r_{1}>r_{2}$ setting $r_{1}=r_{m}$ and $r_{2} \in\left[r_{m}+\frac{\left\|\vec{r}_{C f C i}\right\|}{2 n}, r_{M}\right]$.

If $r_{1}>r_{2}$, two candidate trajectories for the TDV can be considered because there exist two reference arcs having the same $r_{r}$ and satisfying the feasibility condition. One of two candidates has the same properties as the TDV trajectory when $\operatorname{sgn}(\dot{\psi})>0$, and the other candidate has the same properties as the TDV trajectory when $\operatorname{sgn}(\dot{\psi})<0$. Let $n \in\left\{n \in \mathbb{N} \mid n \geq n_{m}\right\}$, and let $r_{1}=r_{M}$. Let $s_{\mathcal{B} n+}$ and $s_{\mathcal{B} n-}$ represent the length $s_{\mathcal{B}}$ of $\mathcal{B}$ when $r_{2}=r_{m}$ and $\operatorname{sgn}(\dot{\psi})>0$, and when $r_{2}=r_{m}$ and $\operatorname{sgn}(\dot{\psi})<0$, respectively. Let $s_{\mathcal{B} n_{S}}$ represent the length $s_{\mathcal{B}}$ of $\mathcal{B}$ when $r_{2}=r_{M}-\frac{\left\|\vec{r}_{C f C i}\right\|}{2 n}$. Since the reference arc is a straight line if $r_{2}=r_{M}-\frac{\left\|\vec{r}_{C f C i}\right\|}{2 n}, \delta_{1}$ and $\delta_{r}$ are equal to 0 from the definition of $\delta_{1}$ and $\delta_{r}$. Then $s_{\mathcal{B} n_{S}}$ is given by:

$$
s_{\mathcal{B} n_{S}}= \begin{cases}\pi\left[(2 n-1) r_{M}-\frac{\left\|\vec{r}_{C f C i}\right\|}{2}\right]+\left(\left|\psi_{1}-\psi_{I}\right|+\left|\psi_{f}-\psi_{2 n}\right|\right) r_{M} & \text { if } \quad r_{1}>r_{2} \\ \pi\left[(2 n-1) r_{m}+\frac{\left\|\vec{r}_{C f C i}\right\|}{2}\right]+\left(\left|\psi_{1}-\psi_{I}\right|+\left|\psi_{f}-\psi_{2 n}\right|\right) r_{m} & \text { if } \quad r 1<r_{2}\end{cases}
$$

Since $r_{r}$ is varied as $r_{2}$ is varied on $\left[r_{m}, r_{M}-\frac{\left\|\vec{r}_{C f C i}\right\|}{2 n}\right]$, we can compare $\arctan \left(-\frac{h_{0}}{s_{\mathcal{B} n+}}\right)$, $\arctan \left(-\frac{h_{0}}{s_{\mathcal{B} n-}}\right)$, and $\arctan \left(-\frac{h_{0}}{s_{\mathcal{B} n_{S}}}\right)$. The case where $r_{1}<r_{2}$ is the same as the case where $r_{1}>r_{2}$ setting $r_{2} \in$ $\left[r_{m}+\frac{\left\|\vec{r}_{C f C i}\right\|}{2 n}, r_{M}\right]$. Therefore, we can assume that the flight path angle $\gamma$ in $\mathcal{B}$ for each $n \in\left\{n \in \mathbb{N} \mid n \geq n_{m}\right\}$ is on $\left[\gamma_{\mathcal{B}_{m}}, \gamma_{\mathcal{B}_{M}}\right]$ where $\gamma_{\mathcal{B}_{m}}$ and $\gamma_{\mathcal{B}_{M}}$ are determined from $\arctan \left(-\frac{h_{0}}{s_{\mathcal{B}}}\right)$ for $s_{\mathcal{B}} \in\left\{s_{\mathcal{B} n+}, s_{\mathcal{B} n-}, s_{\mathcal{B} n_{S}}\right\}$.

Table 1 shows the algorithm to generate the 3D Turning Dubins Vehicle Trajectory. Let $n=n_{m}, r_{1}=r_{M}$, and $r_{2}=r_{m}$. Then $\gamma_{\mathcal{B}_{m}}$ and $\gamma_{\mathcal{B}_{M}}$ are determined by computing $s_{\mathcal{B} n+}, s_{\mathcal{B} n-}$, and $s_{\mathcal{B} n_{S}}$. If the minimum and maximum of flight path angle $\gamma$ in $\mathcal{B}$ for $n$ are less than the minimum flight path angle of the TDV at the initial altitude, then the number $n$ of products of alternating turning arcs is increased because $s_{\mathcal{B}}$ is increased as $n \in\left\{n \in \mathbb{N} \mid n \geq n_{m}\right\}$ is increased. If the interval $\left[\gamma_{\mathcal{B}_{m}}, \gamma_{\mathcal{B}_{M}}\right]$ has an intersection with the constraint $\left[\gamma_{m}, \gamma_{M}\right]$ of the flight path angle of the TDV at $h_{0}, r_{2}$ is increased from $r_{m}$ to $r_{M}-\frac{\left\|\vec{r}_{C f C i}\right\|}{2 n}$. If the minimum $\gamma_{\mathcal{B}_{m}}$ of the flight path angle in $\mathcal{B}$ for $n$ satisfies the vertical feasibility condition 31, a sequence of alternating turning arcs for the TDV is generated by setting $\gamma_{\mathcal{B}_{m}}$ as the adequate flight path angle of the TDV at $h_{0}$, and thus the TDV can reach the landing runway. If $\gamma_{M}$ is less than $\gamma_{\mathcal{B}_{m}}$, feasible and closer runways should be selected despite the safety decrease of landing because the necessary altitude change from the initial to the final (landing) state cannot be achieved within the distance between two centers of the initial and final circular curves.

\section{Compact Flight Envelope Database Generation}

When a damage or failure situation first occurs, an adaptive or emergency flight management system must rapidly respond. First, the autopilot will stabilize the aircraft, ideally at a state close to that commanded for the nominal flight plan, or alternatively at any state that prevents loss-of-control. Simultaneously, system 


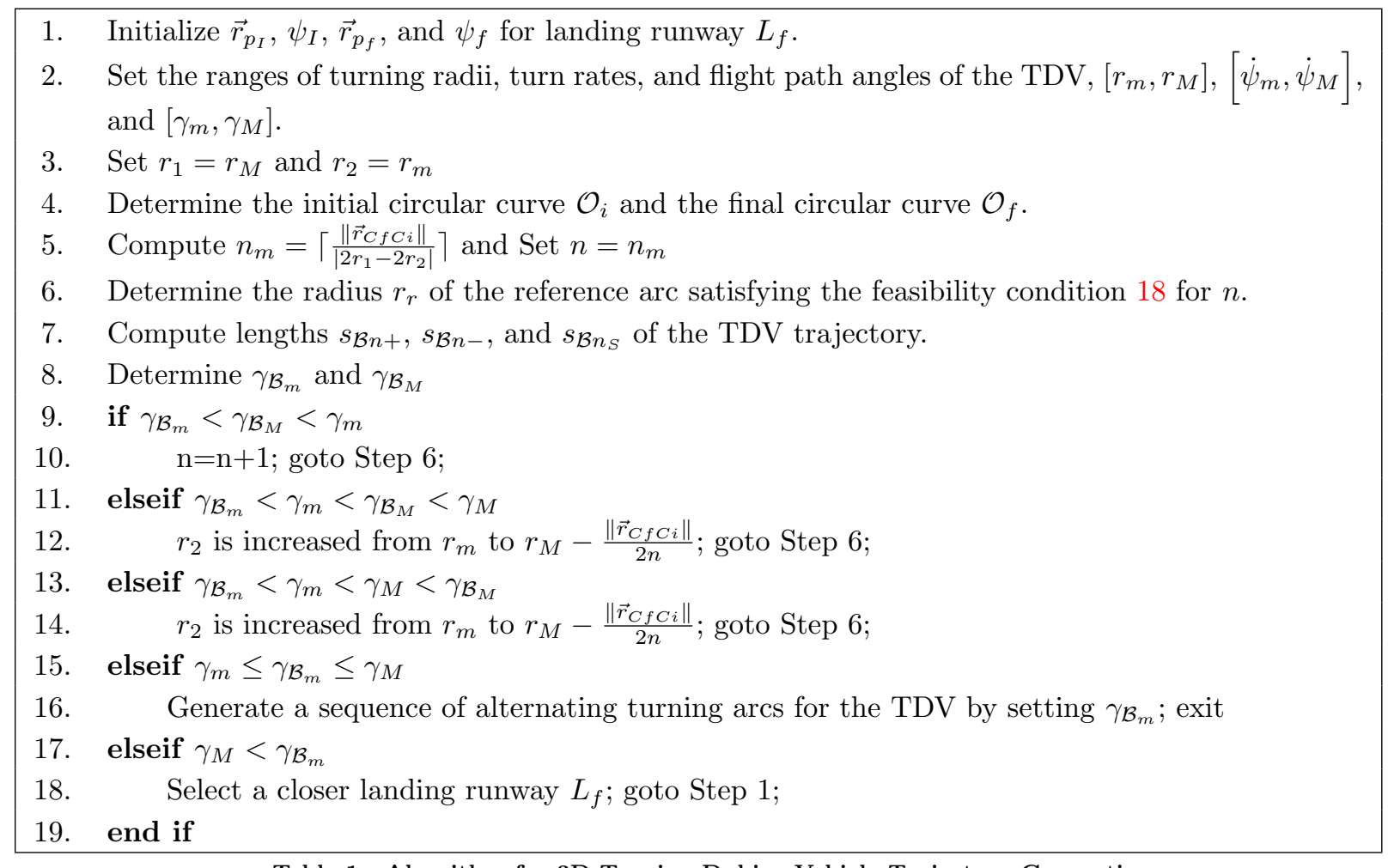

Table 1. Algorithm for 3D Turning Dubins Vehicle Trajectory Generation

identification will generate an envelope estimate that is valid at least locally. System identification can take two basic forms. In cases of actuation failures, sensors may directly indicate the problem. System identification can establish the specific problem characteristics (e.g., loss of thrust results in zero thrust coefficient values). The second case is that the failure is not directly characterizable, as with structural damage; a process such as trim state discovery described above is then required.

Suppose an actuator fails in a constant configuration (e.g., jam). This failure can be identified, and falls into the class of failures that could be predicted thus modeled offline. Each specific jam condition, however, changes the characteristics of the flight envelope to an extent. Trim databases such as those defined in Strube et $\mathrm{al}^{2,3}$ can be generated for a control surface jammed at a particular angle, for example. However, it would be impractical to store different databases for each possible deflection jam angle over the continuous interval of possible values. Using a GUI-based Matlab tool to generate and examine trim databases for known failures (Figure 6), we explored the use of databases that represent the intersection or overlap between stabilizable trim states for different F-16 actuator jam angles. Figures 7 through 9 show trim state sets for F-16 aileron and rudder jams that are stabilizable over the specified interval. Although transitions between these states would be influenced by the specific deflection angle, fundamental properties of the transitions (e.g., settling times) will be comparable given common dynamics and control law formulation. As shown, the F-16 flight envelope is more sensitive to the jam angle value for the aileron than for the rudder. Additionally, as previously discussed, more stabilizable trim states are available at low altitudes than at high altitudes due to the ability to impart higher-magnitude aerodynamic forces and moments thus actuation forces/torques at higher atmospheric densities. As shown in Figure 9, a single envelope can be stored for all rudder deflections from -30 to 30 degrees. As shown in Figure 7, it will be advantageous to store a minimum of three aileron database jam intervals, less than $-10^{\circ},\left[-10^{\circ} 10^{\circ}\right]$, and greater than $10^{\circ}$. As shown in all three figures, altitude also strongly impacts flight envelope extent, although in all examined cases high-altitude flight envelope is a subset of low-altitude flight envelope. Therefore, for jam deflections it will be useful to store databases for multiple altitude intervals, with [0 $10000 \mathrm{ft}],[1000020000 \mathrm{ft}]$ and [20000 $30000 \mathrm{ft}] \mathrm{a}$ sufficient set for F-16 aileron and rudder jams.

In the context of adaptive flight planning, not all data from the detailed flight envelope database need be accessed in real-time to successfully plan a flight. As described in our previous work, ${ }^{2,4}$ a subset of representative data is sufficient to build a flight plan. For a Dubins path, trim states representing straight, 




Figure 6. GUI Interface to Plot Trim States

left, and right turns are required, ideally with level and descending flight path angle options (and possibly climb given terrain requiring an initial climb). For a Turning Dubins Vehicle (TDV) trajectory, bounds on left or right turning radius are required, computable from turn rate and airspeed of stabilizable trim states. Examples of each with trim states selected from control surface jam databases are provided below.

\section{Example Landing Trajectories Possible with the 3D Dubins and 3D TDV Solutions}

Emergency landing trajectories are generated by the Adaptive Flight Planner (AFP) using a trim state set from integrated trim databases or trim state discovery. Suppose an $-10^{\circ}$ aileron jam for F-16 occurs at altitude $10000 \mathrm{ft}$, and that true airspeed is $250 \mathrm{ft} / \mathrm{s}$. Since altitude strongly impacts flight envelope extent, we presume that the AFP uses trim state sets for F-16 aileron jams on an altitude interval $\left[\begin{array}{llll}0 & 10000 \mathrm{ft}\end{array}\right] \mathrm{in}$ Figure 8. At a given altitude $10000 \mathrm{ft}$, the $\mathrm{F}-16$ has a minimum rate $-25 \mathrm{ft} / \mathrm{s}$ of climb and a maximum rate $0 \mathrm{ft} / \mathrm{s}$ of climb, and turn rates between $[-10 \mathrm{ft} / \mathrm{s}-7.5 \mathrm{ft} / \mathrm{s}]$ and $[7.5 \mathrm{ft} / \mathrm{s} 10 \mathrm{ft} / \mathrm{s}]$ can be achieved. The AFP generates a Turning Dubins Vehicle (TDV) trajectory as an emergency landing trajectory rather than a Dubins path because the F-16 cannot maintain straight and/or level flight due to the $-10^{\circ}$ aileron jam, at least given an initial discovery step of the local envelope (rather than the full database over which to plan, which reveals that reduced airspeed can enable straight flight). Suppose the F-16 is located at $40.64^{\circ} N$ and $-73.76^{\circ} \mathrm{W}$ with a heading $210^{\circ}$, and suppose a Landing Site Search (LSS) module in the AFP selects as its top choice JFK $31 \mathrm{~L}\left(40.6398^{\circ} \mathrm{N},-73.7789^{\circ} \mathrm{W}\right)$. Figures 10 and 11 represent Turning Dubins Vehicle trajectories for this emergency satisfying the horizontal and vertical feasibility conditions 18 and 31, and thus the F-16 can reach the JFK 31L runway. Although the minimum values $n_{m}$ of $n$ in $\mathcal{B}$ over $a_{r}$ are 5 and 8 in Figures 10 (a),(b) and Figure 11 (a), respectively, the determined emergency landing trajectories have the increased number 9 and 10 of products of alternating turning arcs to yield the necessary altitude change from the initial state to the final (landing) state. The radius $r_{2}$ of the second circular curve $\mathcal{O}_{2}$ is also increased from the minimum turning radius $1430.2219 \mathrm{ft}$ to $1586.0619 \mathrm{ft}$, and the chosen flight path angle $-5.7361^{\circ}$ falls between $\left[-5.7391^{\circ}-0.5730^{\circ}\right]$ limits to satisfy the vertical feasibility condition 31 , as shown in Figures 10 (c) and (d), although it falls in the steeper range, a problem addressable in future work by increasing the number of turning arc sequences. In Figure 11 for $\operatorname{sgn}(\dot{\psi})>0$ and $r_{1}>r_{2}$, the radius $r_{2}$ of the second circular curve $\mathcal{O}_{2}$ is decreased from the maximum turning radius $1906.9626 \mathrm{ft}$ to $1807.5865 \mathrm{ft}$, and the determined flight path angle is equal to $-5.3304^{\circ}$, again steep but feasible. 


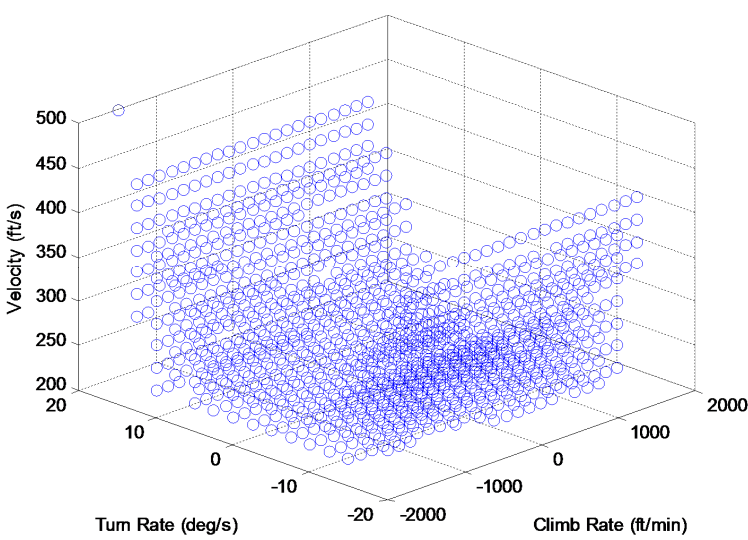

(a) Aileron Jam from $-10^{\circ}$ to $10^{\circ}$ at $0 \mathrm{ft}$

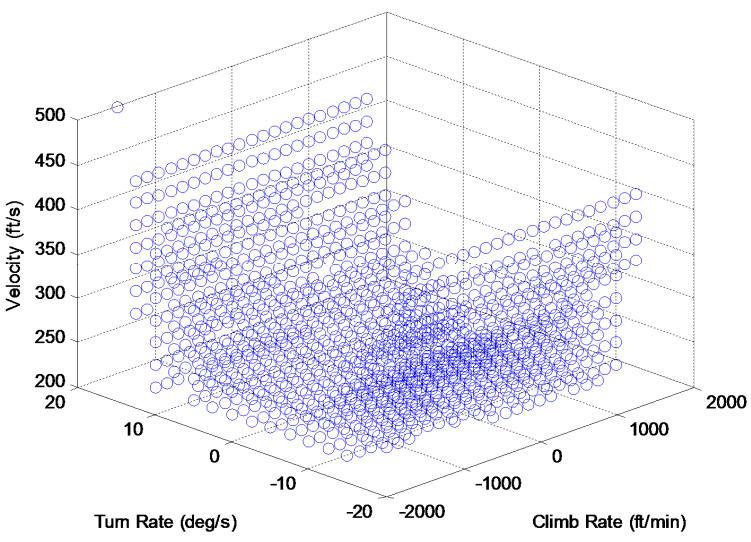

(c) Aileron Jam from $-10^{\circ}$ to $10^{\circ}$ at $10000 \mathrm{ft}$

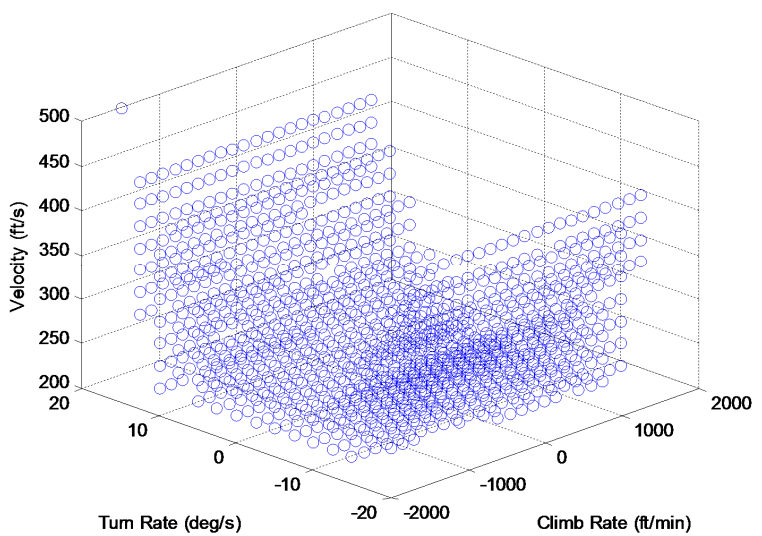

(b) Aileron Jam from $-20^{\circ}$ to $10^{\circ}$ at $10000 \mathrm{ft}$

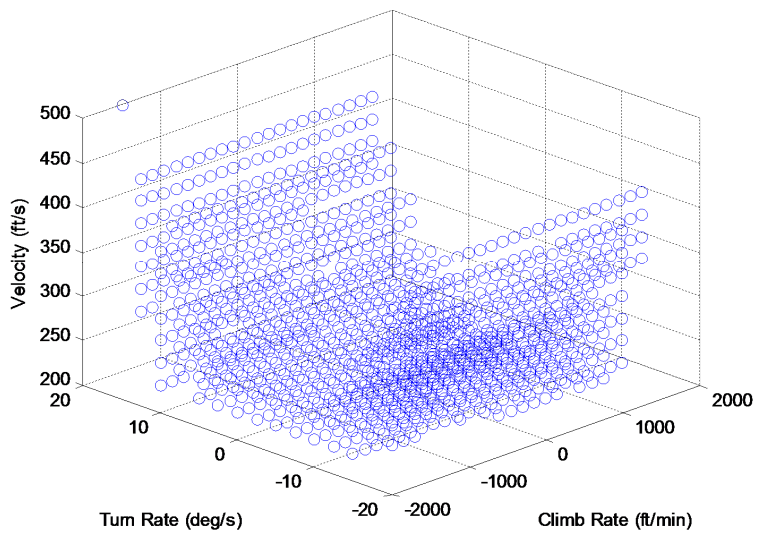

(d) Aileron Jam from $-10^{\circ}$ to $10^{\circ}$ at $20000 \mathrm{ft}$

Figure 7. Flight Envelopes for Different Jammed Aileron Deflection Intervals

Suppose the F-16 has a true airspeed $225 \mathrm{ft} / \mathrm{s}$ about the same aileron jam and the same altitude as the previous emergency. From the trim state set for F-16 aileron jams over altitude interval [010000ft], the F-16 has a range $[-25 \mathrm{ft} / \mathrm{s} 0 \mathrm{minft} / \mathrm{s}]$ of climb rate if turn rate for the $\mathrm{F}-16$ is on $[-7.5 \mathrm{ft} / \mathrm{s}-2.5 \mathrm{~min} \mathrm{ft} / \mathrm{s}]$ and $[2.5 \mathrm{ft} / \mathrm{s} 7.5 \mathrm{minft} / \mathrm{s}]$, and a range $[-25 \mathrm{ft} / \mathrm{s} 0 \mathrm{minft} / \mathrm{s}]$ of climb rate if turn rate for the $\mathrm{F}-16$ is on $[-7.5 \mathrm{ft} / \mathrm{s} 7.5 \mathrm{minft} / \mathrm{s}]$ at a given altitude $10000 \mathrm{ft}$. The AFP can generate a Dubins trajectory and a TDV trajectory for emergency landing because the damaged F-16 has a rate of turn where the F-16 can fly straight. Suppose the F-16 in an emergency is located at $40.69^{\circ} \mathrm{N}$ and $73.72^{\circ} \mathrm{W}$ with a heading $210^{\circ}$. The LSS identifies 136 feasible landing runways and selects as its top choice JFK 31L. However, the AFP generates a Dubins path to the JFK 22R runway to guarantee sufficient altitude change between the given initial position and the landing runway ${ }^{1}$ by extending final approach to the runway, as shown in Figure 12. If the tangent segment of a Dubins path has a sufficient length to generate a direct (Dubins) trajectory from an initial position of the aircraft to the top-ranked runway, the AFP generates a Dubins path without extending final approach to the runway. If the length of the tangent segment is sufficiently long, and the flight path angle of the tangent segment in a Dubins path is less than the minimum flight path angle from the used trim state set at a given altitude, the AFP constructs the "S-turn"solution, i.e. a Dubins path inserting an intermediate turn, ${ }^{1}$ as shown in Figure 13. In Figure 13, the F-16 has the same conditions as the case of a direct (Dubins) trajectory except for the F-16 location $\left(40.68^{\circ} \mathrm{N},-73.82^{\circ} \mathrm{W}\right)$ and the use of runway JFK 31L.

For the case of the F-16 with a true airspeed $225 \mathrm{ft} / \mathrm{s}$, the AFP can construct a Dubins path and a TDV trajectory at the given altitude $10000 \mathrm{ft}$ due to a range of turn rate, $[-7.5 \mathrm{ft} / \mathrm{s} 7.5 \mathrm{minft} / \mathrm{s}]$. Suppose the 


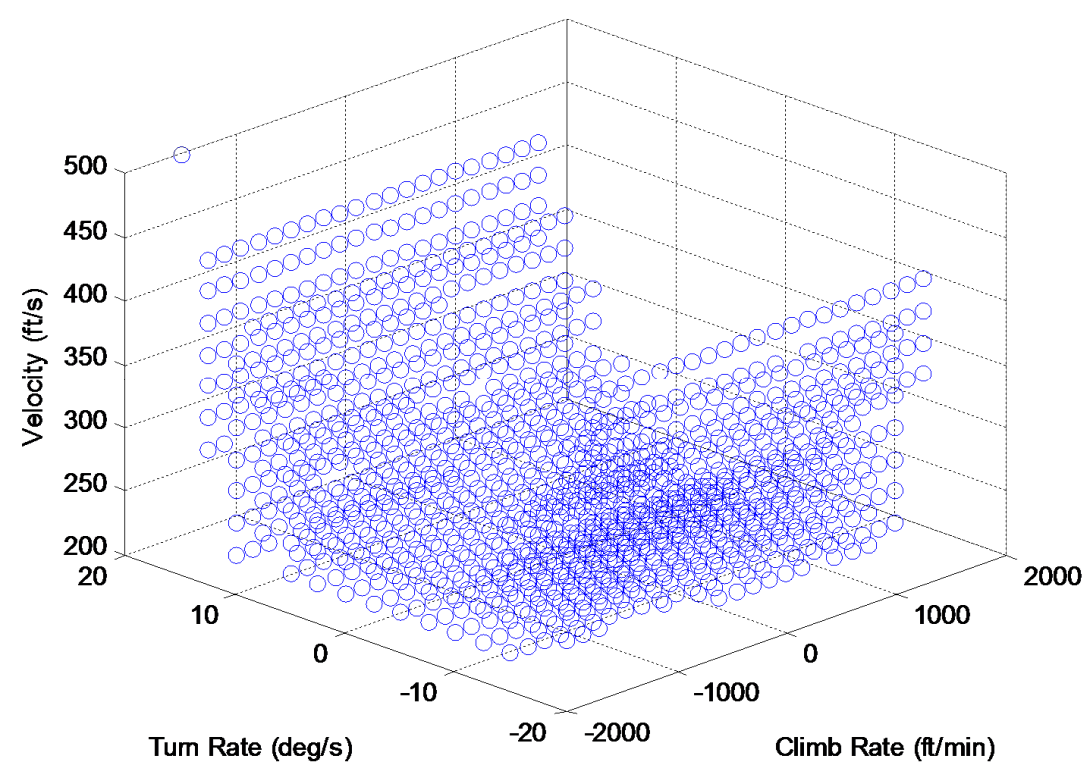

Figure 8. Flight Envelope for Aileron Jam over an Altitude Interval

F-16 has the same conditions as the case of a direct (Dubins) trajectory, but turn rate for the F-16 is on $[-7.5 \mathrm{ft} / \mathrm{s}-2.5 \mathrm{minft} / \mathrm{s}]$ and $[2.5 \mathrm{ft} / \mathrm{s} 7.5 \mathrm{minft} / \mathrm{s}]$. From Figure 8 , the $\mathrm{F}-16$ has a range $[-25 \mathrm{ft} / \mathrm{s} 0 \mathrm{minft} / \mathrm{s}]$ in climb rate. Unlike the case of a direct (Dubins) trajectory, the TDV trajectory generated by the AFP can reach runway JFK 31L that the Landing Site Search (LSS) module selected as its top-priority runway, and two feasibility conditions of the TDV are satisfied to guarantee a successful emergency landing, as shown in Figures 14 and 15. The vertical feasibility condition 31 is satisfied by the flight path angle $\gamma_{\mathcal{B}_{m}}$ in $\mathcal{B}$ for the case where $r_{1}=r_{M}=5148.6315 \mathrm{ft}, r_{2}=r_{m}=1716.2105 \mathrm{ft}$, and $n=n_{m}=4$ in Figure 14 and $n=n_{m}=5$ in Figure 15. Note that the determined flight path angle is $-6.0143^{\circ}$ in Figure 14 and $-5.6006^{\circ}$ in Figure 15.

\section{Conclusions and Future Work}

We have presented an adaptive flight planning architecture capable of autonomously managing situations in which aircraft performance is degraded due to damage or system failures. Our flight planner first identifies or discovers the degraded performance characteristics by exploring the space of climb rate, turn rate, and airspeed values in a manner that ensures the aircraft remains stabilizable but that determines a set of feasible trim states to sequence into an emergency landing plan. Based on the identified envelope properties, either a Dubins or Turning Dubins Vehicle flight plan is generated, providing a comprehensive real-time trajectory planning tool that ultimately can be certified due to its use of geometry that ultimately can be proven correct and complete. We have presented integrated flight envelopes, results from trim state discovery, and a series of Dubins and TDV flight plans for an F-16 experiencing rudder and aileron jam failures.

In future work we will apply this approach to different and more challenging damage models and will integrate our flight planner within the full emergency flight management framework. In the TDV trajectories, the reference arcs may be undefined as $n$ and $r_{2}$ are varied in order to satisfy the vertical feasibility condition although the TDV trajectories satisfy the horizontal feasibility condition for the minimum number of products of alternating turning arcs. Specifically, if the distance between two centers of the initial and final circular curves is too close, and if the difference between two turning radii is too small, the reference arcs might be undefined as the number of products of alternating turning arcs and the radius of the second circular curve are changed when the AFP strives to generate a TDV flight plan as an emergency landing trajectory. In future work, the case of the undefined reference arc must be managed, and thus an intermediate turn will be inserted analogous to the "S-turn" solution from our Dubins path method. ${ }^{1}$ 


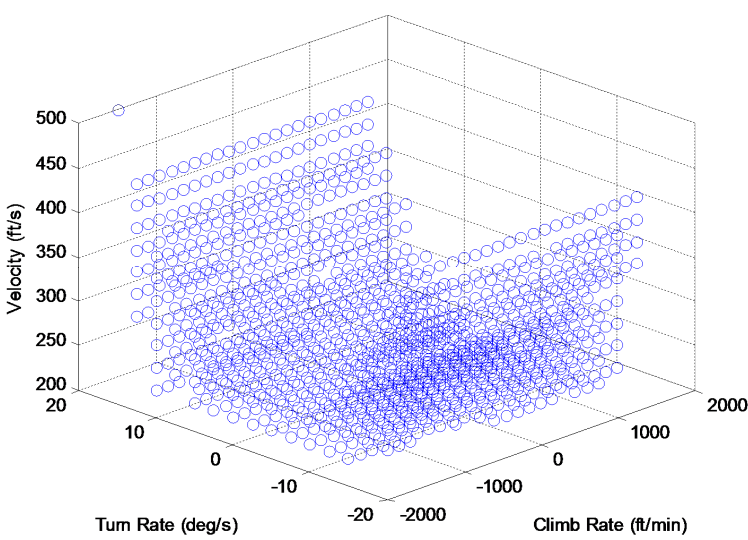

(a) Rudder Jam from $-30^{\circ}$ to $30^{\circ}$ at $0 \mathrm{ft}$

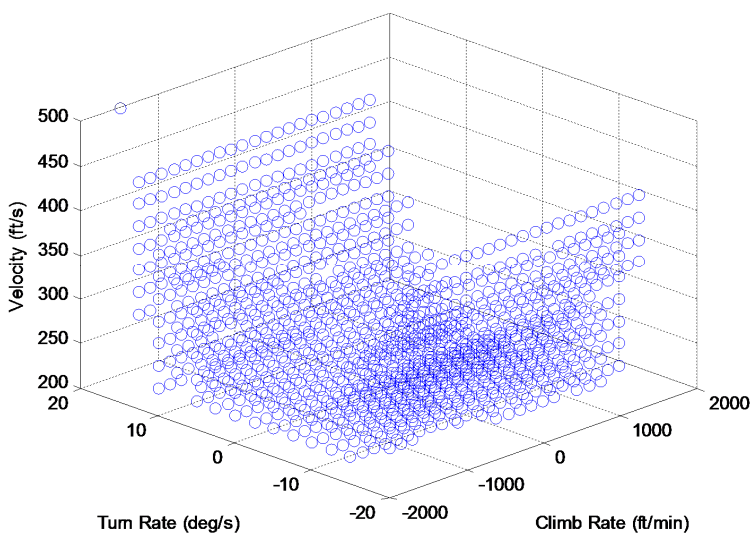

(c) Rudder Jam from $-30^{\circ}$ to $30^{\circ}$ at $20000 \mathrm{ft}$

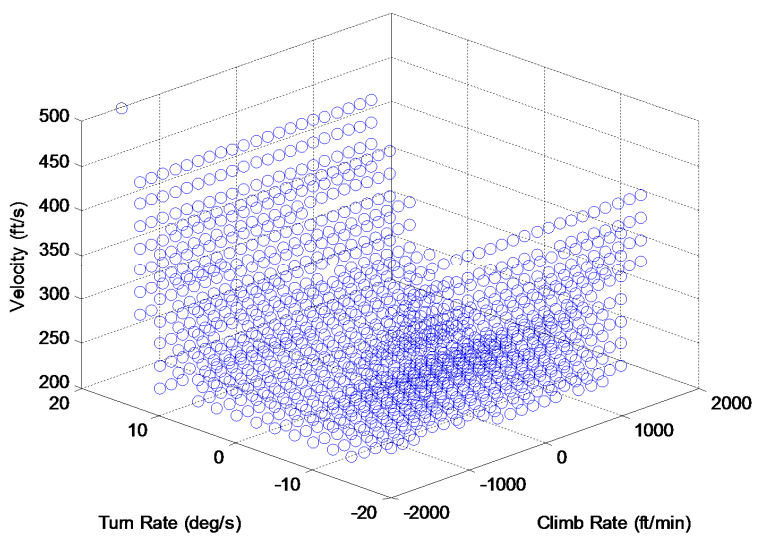

(b) Rudder Jam from $-30^{\circ}$ to $30^{\circ}$ at $10000 \mathrm{ft}$

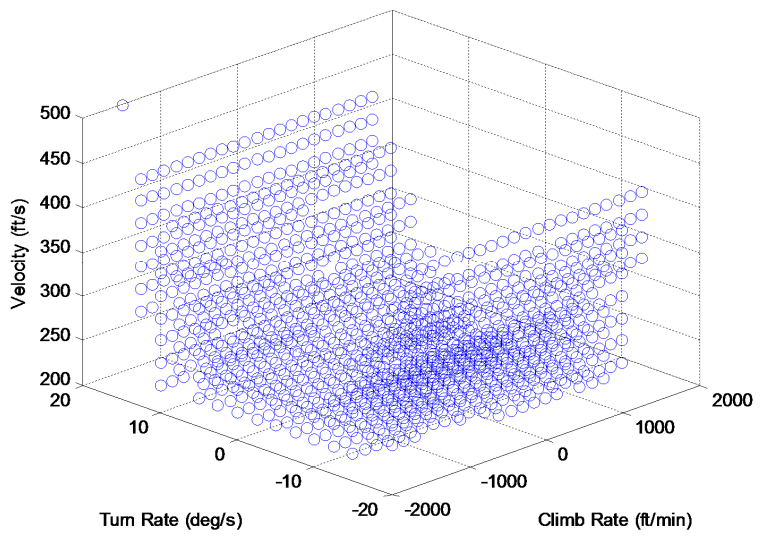

(d) Rudder Jam from $-30^{\circ}$ to $30^{\circ}$ at $0 \mathrm{ft}$ to $20000 \mathrm{ft}$

Figure 9. Flight Envelopes for Different Jammed Rudder Deflection Intervals

\section{Acknowledgments}

The authors would like to thank collaborator Robert Sanner from the University of Maryland for his valuable insights and contributions to the adaptive flight planner. This research was supported in part under the NASA Aviation Safety program, Integrated Resilient Aircraft Control (IRAC) NRA Cooperative Agreement NNX08AB93A.

\section{References}

${ }^{1}$ Atkins, E., Portillo, A., and Strube, M., "Emergency Flight Planning applied to Total Loss of Thrust," Journal of Aircraft, Vol. 43, No. 4, Jul.-Aug. 2006, pp. 1205-1216.

${ }^{2}$ Strube, M., Sanner, R., and Atkins, E., "Dynamic Flight Guidance Recalibration after Actuator Failure," Proceedings of the 1st AIAA Intelligent Systems Technical Conference, Chicago, Illinois, Sep. 2004.

${ }^{3}$ Strube, M. J., Post-failure Trajectory Planning From Feasible Trim State Sequences, Master's thesis, Aerospace Engineering Dept., University of Maryland, College Park,, 2005.

${ }^{4}$ Tang, Y., Atkins, E., and Sanner, R., "Emergency Flight Planning for a Generalized Transport Aircraft with Left Wing Damage," Proceedings of the AIAA Guidance, Navigation, and Control Conference, AIAA, Hilton Head, South Carolina, Aug. 2007.

${ }^{5}$ Choi, H. and Atkins, E., "An Analytic Trajectory Planner for Aircraft with Severe Damage or Failures," Proceedings of the AIAA Infotech@Aerospace Conference, AIAA, Seattle, Washington, Apr. 2009.

${ }^{6}$ Yi, G. and Atkins, E., "Trim State Discovery for an Adaptive Flight Planner," Proceedings of the 48th AIAA Aerospace Science Meeting, AIAA, Orlando, Florida, Jan. 2010. 


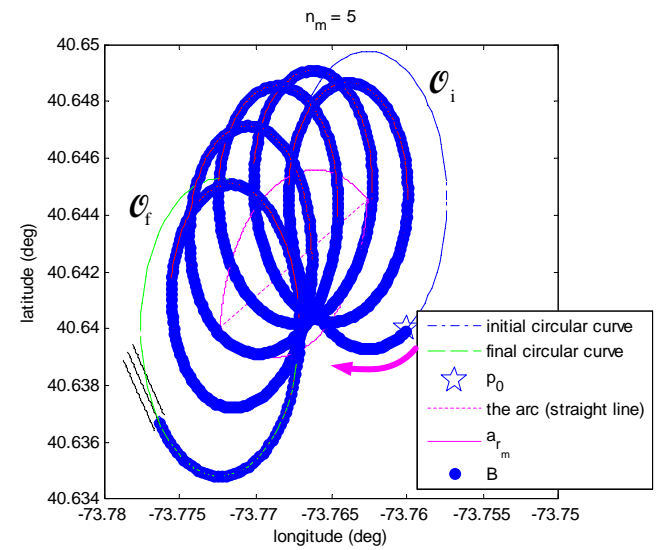

(a) 2D TDV Trajectories for $n_{m}$

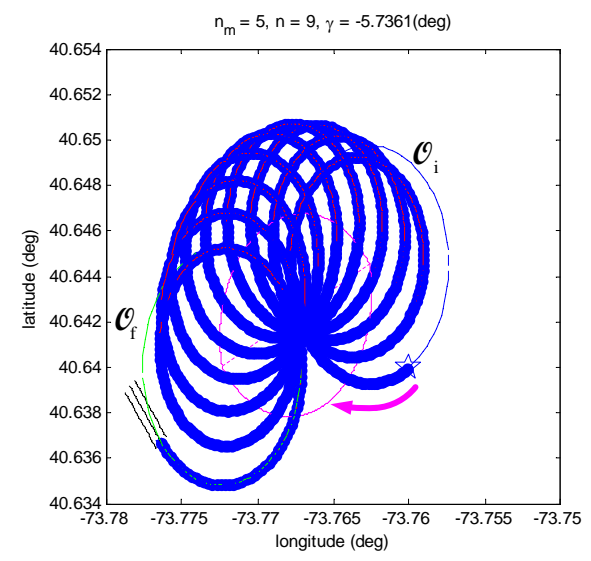

(c) 2D TDV Trajectory Satisfying Feasibility Conditions

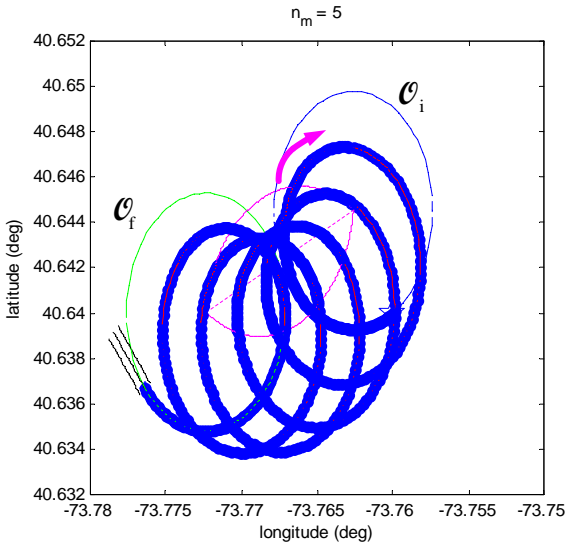

(b) 2D TDV Trajectories for $n_{m}$

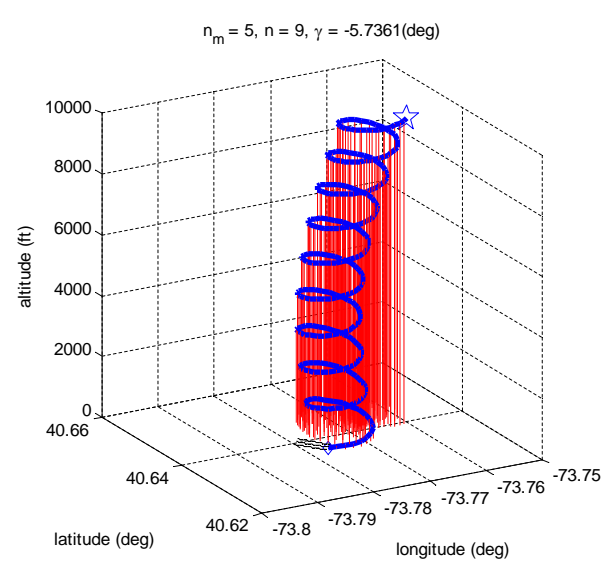

(d) 3D TDV Trajectory Satisfying Feasibility Conditions

Figure 10. TDV Trajectory to the JFK 31L for a $-10^{\circ}$ Aileron Jam of the F-16 with an True Airspeed $250 \mathrm{ft} / \mathrm{s}$ if $\operatorname{sgn}(\dot{\psi})<0$ and $r_{1}>r_{2}$

${ }^{7}$ Stevens, B. and Lewis, F., Aircraft Control and Simulation, Wiley and Sons, 1992.

${ }^{8}$ Savla, K., Frazzoli, E., and Bullo, F., "Traveling Salesperson Problems for the Dubins Vehicle," Automatic Control, IEEE Transactions on, Vol. 53, No. 6, july 2008, pp. $1378-1391$.

${ }^{9}$ Michel, A., Nam, B., and Vittal, V., "Computer generated Lyapunov functions for interconnected systems: Improved results with applications to power systems," Circuits and Systems, IEEE Transactions on, Vol. 31, No. 2, Feb. 1984, pp. 189 $-198$.

${ }^{10}$ Vannelli, A. and Vidyasagar, M., "Maximal lyapunov functions and domains of attraction for autonomous nonlinear systems," Automatica, Vol. 21, No. 1, 1985, pp. $69-80$.

${ }^{11}$ Chesi, G., Garulli, A., Tesi, A., and Vicino, A., "LMI-based Computation of Optimal Quadratic Lyapunov Functions for Odd Polynomial Systems," International Journal of Robust and Nonlinear Control, Vol. 15, No. 1, 2005, pp. 35-49.

${ }^{12}$ Kohout, B., "Challenges in Real-Time Obstacle Avoidance," AAAI Spring Symposium on Real-Time Autonomous Systems, AAAI, Palo Alto, California, Mar. 2000.

${ }^{13}$ Lumelsky, V. and Skewis, T., "Incorporating range sensing in the robot navigation function," Systems, Man and Cybernetics, IEEE Transactions on, Vol. 20, No. 5, sep/oct 1990, pp. $1058-1069$.

${ }^{14}$ Lumelsky, V. J. and Stepanov, A. A., "Path-planning strategies for a point mobile automaton moving amidst unknown obstacles of arbitrary shape," Autonomous robot vehicles, 1990, pp. 363-390.

${ }^{15}$ Khatib, O., "Real-Time Obstacle Avoidance for Manipulators and Mobile Robots," The International Journal of Robotics Research, Vol. 5, No. 1, 1986, pp. 90-98.

${ }^{16}$ Reddy, E. R., Kuderu, R., and L., K. P., "Robots with Obstacles," Research Journal of Applied Sciences, Vol. 2, No. 12, 2007, pp. 1218-1222.

${ }^{17}$ Bruce, H. G., The Elements of Geometry, BiblioBazaar, LLC, 2009. 


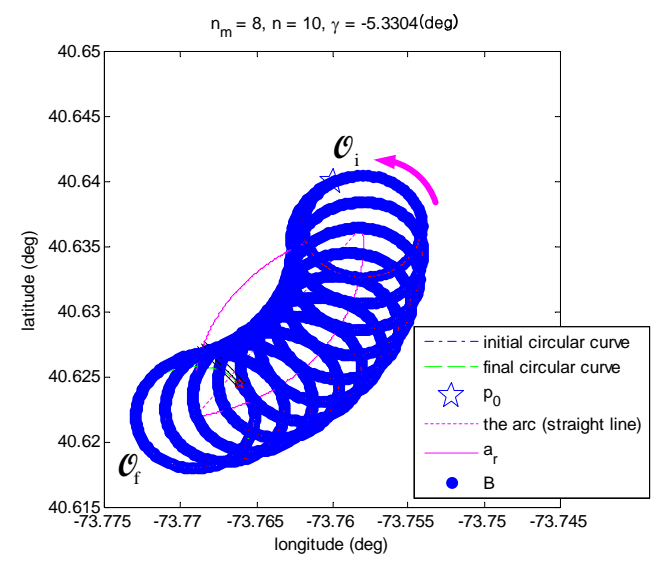

(a) 2D TDV Trajectory Satisfying Feasibility Conditions

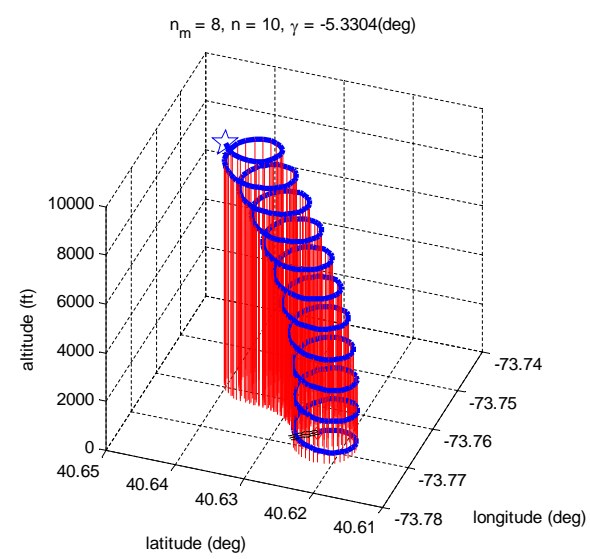

(b) 3D TDV Trajectory Satisfying Feasibility Conditions

Figure 11. TDV Trajectory to the JFK 31L for a $-10^{\circ}$ Aileron Jam of the F-16 with an True Airspeed $250 \mathrm{ft} / \mathrm{s}$ if $\operatorname{sgn}(\dot{\psi})>0$ and $r_{1}<r_{2}$



(a) 2D Dubins Trajectory

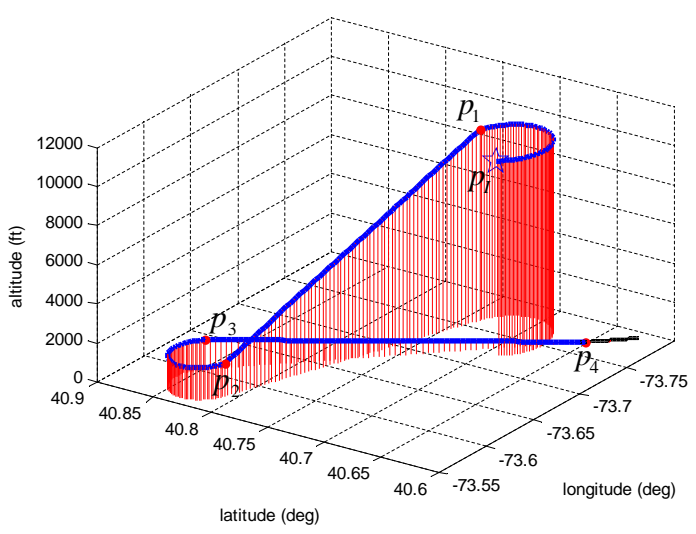

(b) 3D Dubins Trajectory

Figure 12. Direct Dubins Trajectory to the JFK 22R for a $-10^{\circ}$ Aileron Jam of the F-16 with an True Airspeed 225 $\mathrm{ft} / \mathrm{s}$

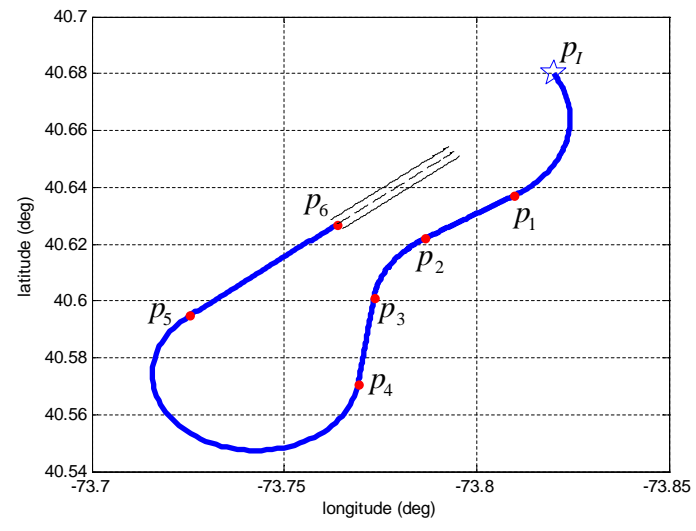

(a) 2D Dubins Trajectory

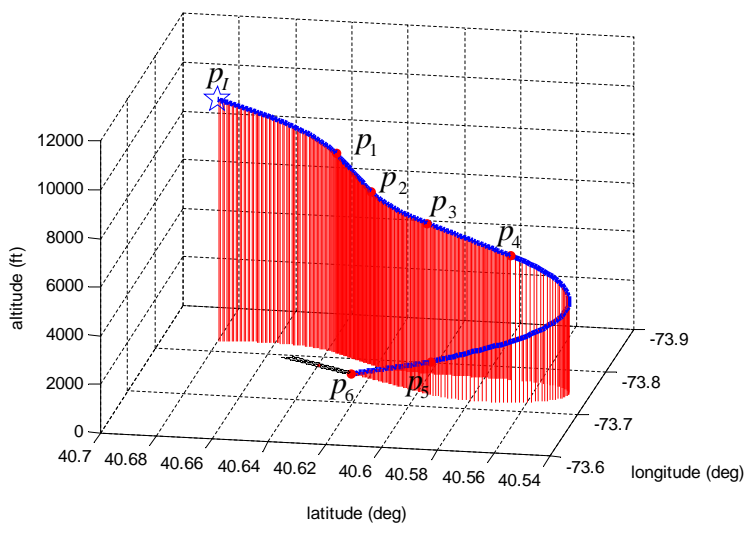

(b) 3D Dubins Trajectory

Figure 13. S-turn Dubins Trajectory to the JFK 31L for a $-10^{\circ}$ Aileron Jam of the F-16 with an True Airspeed 225 $\mathrm{ft} / \mathrm{s}$ 




(a) 2D Dubins Trajectory Satisfying Feasibility Conditions

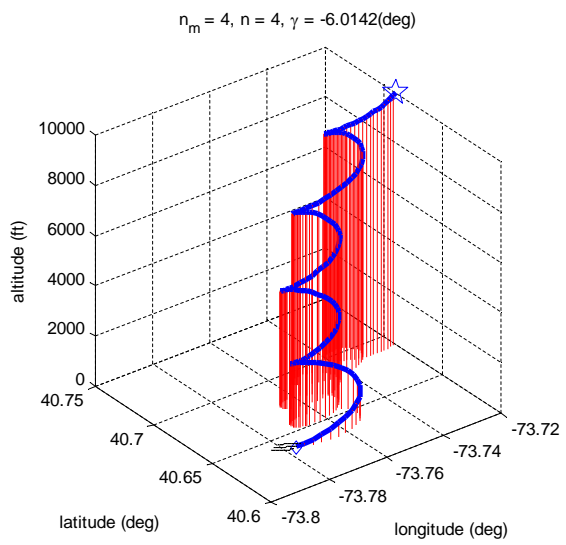

(b) 3D Dubins Trajectory Satisfying Feasibility Conditions

Figure 14. TDV Trajectories to the JFK 31L for a $-10^{\circ}$ Aileron Jam of the F-16 with an True Airspeed $225 \mathrm{ft} / \mathrm{s}$ if $\operatorname{sgn}(\dot{\psi})<0$ and $r_{1}>r_{2}$

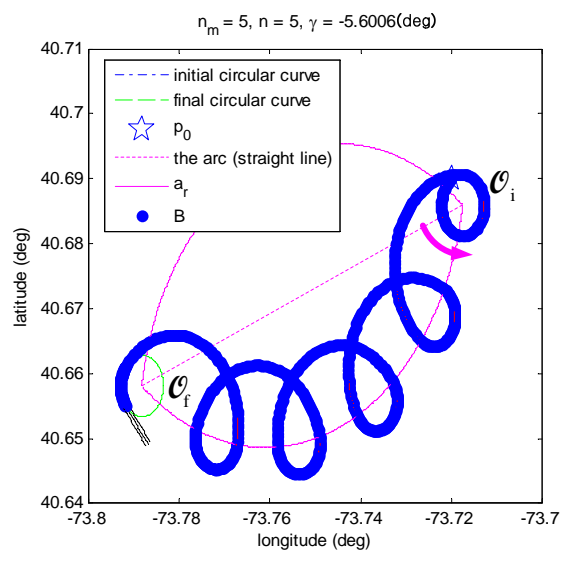

(a) 2D Dubins Trajectory Satisfying Feasibility Conditions

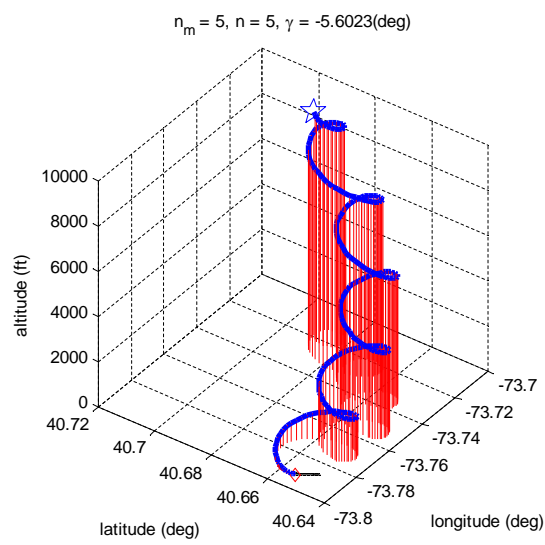

(b) 3D Dubins Trajectory Satisfying Feasibility Conditions

Figure 15. TDV Trajectories to the JFK 31L for a $-10^{\circ}$ Aileron Jam of the F-16 with an True Airspeed $225 \mathrm{ft} / \mathrm{s}$ if $\operatorname{sgn}(\dot{\psi})>0$ and $r_{1}<r_{2}$ 